\title{
Explicit finite deformation analysis of isogeometric membranes
}

Lei Chen ${ }^{1}$, Nhon Nguyen-Thanh ${ }^{2}$, Hung Nguyen-Xuan ${ }^{3}$, Timon Rabczuk ${ }^{2,4}$, Stéphane Pierre Alain Bordas ${ }^{5}$ and Georges Limbert ${ }^{1,6, *}$

${ }^{1}$ national Centre for Advanced Tribology at Southampton (nCATS), Engineering Sciences, Faculty of Engineering and the Environment, University of Southampton, Southampton SO17 1BJ, UK

${ }^{2}$ Institute of Structural Mechanics, Bauhaus-University Weimar, Marienstrasse 15, 99423 Weimar, Germany

${ }^{3}$ Faculty of Mathematics and Computer Science, University of Science, Vietnam National University-Ho Chi Minh,Vietnam

4 School of Civil, Environmental and Architectural Engineering, Korea University, 145, Anam-ro, Seongbuk-gu, Seoul, 136-701, Korea

${ }^{5}$ Cardiff School of Engineering, Institute of Modelling \& Simulation in Mechanics \& Materials, Cardiff University, Queen's Buildings, The Parade, Cardiff CF24 3AA, Wales, UK

${ }_{6}$ Bioengineering Science Research Group, Engineering Sciences, Faculty of Engineering and the Environment, University of Southampton, Southampton SO17 1BJ, UK

\begin{abstract}
NURBS-based isogeometric analysis was first extended to thin shell/membrane structures which allows for finite membrane stretching as well as large deflection and bending strain. The assumed non-linear kinematics employs the Kirchhoff-Love shell theory to describe the mechanical behaviour of thin to ultrathin structures. The displacement fields are interpolated from the displacements of control points only, and no rotational degrees of freedom are used at control points. Due to the high order $C^{k}(k \geq 1)$ continuity of NURBS shape functions the Kirchhoff-Love theory can be seamlessly implemented. An explicit time integration scheme is used to compute the transient response of membrane structures to time-domain excitations, and a dynamic relaxation method is employed to obtain steady-state solutions. The versatility and good performance of the present formulation is demonstrated with the aid of a number of test cases, including a square membrane strip under static pressure, the inflation of a spherical shell under internal pressure, the inflation of a square airbag and the inflation of a rubber balloon. The mechanical contribution of the bending stiffness is also evaluated.
\end{abstract}

Key words: Membrane; Kirchhoff-Love shell; isogeometric; NURBS; explicit; dynamic relaxation.

$\begin{array}{ll}\text { Journal: } & \text { Computer Methods in Applied Mechanics and Engineering } \\ \text { Article type: } & \text { Research Paper } \\ \text { Date: } & \text { Accepted 23 April } 2014\end{array}$

Corresponding author's:

Georges Limbert, g.limbert@soton.ac.uk 


\section{Introduction}

Thin membrane structures are ubiquitous in Nature from the lipid membrane of animal cells, virus shells, wings of flying insects, through the pleura (lung membrane), the vitelline membrane in eggs, the placenta of mammals, to the heart valves and the skin. A key feature of membrane structures is their high slenderness ratio and their ability to resist significant membrane stress whilst minimising their weight [1]. Due to their extreme efficiency in addressing particular types of environments and achieving outstanding functionalities, engineered membrane structures are also used for a wide range of applications such as building fabric structures (e.g. exhibition pavilions, permanent roofing structures), bioprosthetics (e.g. artificial heart valves), biomimetic (e.g. flying robots, artificial jelly-fish propulsion systems) and deployable structures (e.g. airbag, parachute, landing system of Mars Rover) [2, 3].

The large slenderness associated with membrane structures means that the structural bending stiffness is generally very small and even negligible $[1,4]$. In the latter case, stress states characterised by negative principal components are not admissible. As a result, large deformations such as ballooning, local wrinkling or folding are produced to minimise the potential energy of the system [5]

The mechanical response of these very thin shell/membrane structures is more naturally described by the Kirchhoff-Love shell theory [6-8] incorporating membrane strains and change in curvature due to bending rather than full three-dimensional constitutive theories or Reissner-Mindlin shell theory [1] which include transverse shear stress. The accurate modelling of such thin structures under large deflection, rotation and strain is essential for understanding the mechanics of biological membranes (here, membrane refers to the biological meaning) [9-11] which is one of the motivations for the present study. The only practical way to analyse membrane structures featuring complex geometries is to use numerical methods such as the finite element method (FEM) [12-17]. The robust numerical simulation of membrane structures for arbitrary kinematics and non-linear constitutive laws is a challenging research area. Moreover, for practical applications, the design of membrane structures could be significantly streamlined by tying computer-aided design $(\mathrm{CAD})$ and analysis together. Although the situation is rapidly evolving since the visionary work of Hughes et al. [18], engineering design and numerical analysis are traditionally not tightly integrated. The typical situation in engineering practice is that designs are encapsulated in computer-aided design (CAD) systems which are subsequently converted into finite element meshes through a, generally, time-consuming process which can be done at best semi-automatically. It is estimated that about $80 \%$ of overall analysis time is devoted to mesh generation in a number of industries such aerospace or automotive [19].

An important point for the computational analysis of Kirchhoff-Love shells is that $C^{1}$ continuity is required between elements $[6,7,20,21]$ as curvature is a function of the second derivatives of the displacement, which is rather difficult to achieve for free-form geometries when using polynomial basis functions [6]. These polynomial shape functions, when applicable, introduce undesirably high order polynomials with inherent disadvantages such as oscillations in the discrete solution and costly numerical integration (see, e.g. references [20, 21], among many others). The computational burden associated with these approaches is particularly onerous in the presence of strong gradients in the solution and/or in the case of costly stress-update procedures at the quadrature-point level.

Rabzcuk et al. developed mesh-free thin shell element formulations accounting for large deformations and fracture within partition of unity-enrichment [22, 23]. Only the shell mid-surface was discretised (not the director field) and no rotational degrees of freedom were used. Rabzcuk et al. [24] later extended their mesh-free approach to model fracture arising from fluid-structure interactions. Millán et al. [25] developed a robust automatic method based on smooth mesh-free maximum-entropy approximants to circumvent global parameterisation of surfaces of complex geometry and topology. The technique was applied to geometrically-exact thin shell problems. This method was recently extended by Millán et al. [26] to address shortcomings arising from overlapping of patches which resulted in redundant numerical quadrature, and so, computational cost.

Recently, the idea of using non-uniform rational B-Splines (NURBS) [27] as basis functions for analysis was introduced by Hughes et al. [18, 19], and was named isogeometric analysis. In isogeometric analysis, the functions for the geometry description are used as basis functions for the analysis. Thus, the analysis works on a geometrically exact model. This offers the possibility to close the existing gap between design and analysis by merging design geometry and analysis model. It was demonstrated that not only were NURBS applicable to engineering analysis, but that they were better suited for many applications, and were able to deliver accuracy superior to standard finite elements (see, e.g., [28-36]). 
Subdivision surfaces and, more recently, T-Splines [37, 38] and PHT-Splines [39-41], were also successfully employed in isogeometric analysis contexts. More importantly, NURBS are smooth, higher order functions which are used for geometric design and have become standard in CAD (computer aided design) programs. They allow great geometric flexibility and high order continuities at the same time. NURBS are therefore ideally suited as basis functions for Kirchhoff-Love shell [20, 41-43].

Making use of these significant properties, Kiendl et al. [42, 43] developed a NURBS-based isogeometric Kirchhoff-Love shell which can capture both geometrically linear and non-linear deformations. However, only preliminary results were reported in their non-linear analysis. Further, Kiendl et al.'s non-linear analysis was solved using an implicit method. As highlighted earlier in this section, the bending stiffness of membrane structures is in general extremely small and is often assumed to be negligible. In that case, difficulties arise in incremental-type solution procedures, due to the fact that the vanishing bending stiffness leads to an ill-conditioned or even singular stiffness matrix, which renders implicit-based methods difficult or unable to converge [4].

In the present study, a framework for explicit NURBS-based isogeometric analysis for thin to ultra-thin shell/membrane structures, valid for arbitrary kinematics (finite deformations and strains) is developed. Both membrane strains and bending stiffness are considered using the Kirchhoff-Love assumption. The displacement fields are interpolated from the displacements of control points only, and no rotational degrees of freedom are used at control points. Due to the high order $C^{k}(k \geq 1)$ continuity of NURBS shape functions the Kirchhoff-Love theory can be seamlessly implemented. An explicit time integration scheme [44] is used to compute the transient response of the membrane structures to time-domain excitations, and a dynamic relaxation method $[17,45,46]$ is employed to obtain steady-state solutions. The performance of the current isogeometric formulation and implementation is assessed through a series of examples featuring elastic and hyperelastic materials, and also compared to analytical, experimental and "traditional" (i.e. based on Lagrange polynomial interpolation functions) finite element analyses. The influence of the mechanical contribution of the bending stiffness to the general behaviour of the thin shell structure is also assessed.

\section{A brief on NURBS-based isogeometric analysis}

Non-uniform rational B-Splines (NURBS) are a standard tool for describing and modelling curves and surfaces in computer-aided design and graphics (e.g. see Piegl and Tiller [27]). In this section, we give a brief overview of NURBS-based isogeometric analysis (NURBS IGA), for which an extensive account has already been given in Hughes et al. [18], Cottrell et al. [19] and Nguyen et al. [47].

B-Splines are piecewise polynomial curves composed of linear combinations of B-Spline basis functions. The function coefficients are points in space, referred to as control points. A knot vector $\Xi$ is a set of nondecreasing real numbers representing coordinates in the parametric space of the curve:

$$
\Xi=\left\{\xi_{1}, \xi_{1}, \ldots, \xi_{n+p+1}\right\}
$$

where $p$ is the order of the B-Spline and $n$ is the number of basis functions (and control points). The interval $\left[\xi_{1}, \xi_{n+p+1}\right]$ is called a patch. The detailed procedure for constructing B-Splines can be found in the monograph of Piegl and Tiller [27]. However, some important properties are listed as follows:

1. If all knots are equally spaced, the knot vector is called uniform, otherwise, non-uniform;

2. A B-Spline basis function is $C^{\infty}$ continuous inside a knot span, i.e. between two distinct knots, and $C^{p-1}$ continuous at a single knot. A knot value can appear more than one time and is then called a multiple knot. At a knot of multiplicity $k$ the continuity is $C^{p-k}$.

3. If the first and the last knot have multiplicity $p+1$, the knot vector is called open [27]. In a B-Spline with an open knot vector the first and the last control points are interpolated and the curve is tangential to the control polygon at the start and the end of the curve. Such open knot vectors are assumed for the remainder of this text.

Given a knot vector $\Xi$, the B-Spline basis functions are defined recursively starting with $p=0$ (piecewise constants): 


$$
N_{i, 0}(\xi)= \begin{cases}1 & \text { if } \xi_{i} \leq \xi \leq \xi_{i+1} \\ 0 & \text { otherwise }\end{cases}
$$

For $p=1,2,3, \ldots$ the basis is defined by the Cox-de Boor recursion formula:

$$
N_{i, p}(\xi)=\frac{\xi-\xi_{i}}{\xi_{i+p}-\xi_{i}} N_{i, p-1}(\xi)+\frac{\xi_{i+p+1}-\xi}{\xi_{i+p+1}-\xi_{i+1}} N_{i+1, p-1}(\xi)
$$

Using tensor products, B-Spline surfaces can be constructed starting from knot vectors $\Xi=\left\{\xi_{1}, \xi_{1}, \ldots, \xi_{n+p+1}\right\}$ and $\mathrm{I}=\left\{\eta_{1}, \eta_{2}, \ldots, \eta_{n+p+1}\right\}$, and an $n \times m$ net of control points $\mathbf{x}_{i, j}$ also called the control mesh. One-dimensional basis functions $N_{i, p}$ and $M_{j, q}(i=1, \ldots, n ; j=1, \ldots, m)$ of order $p$ and $q$, respectively, are defined from the corresponding knot vectors, and the B-Spline surface is constructed as:

$$
S(\xi, \eta)=\sum_{i=1}^{n} \sum_{j=1}^{m} N_{i, p}(\xi) M_{j, q}(\eta) \mathbf{x}_{i, j}
$$

The patch for the surface is now the domain $\left[\xi_{1}, \xi_{n+p+1}\right] \times\left[\eta_{1}, \eta_{m+q+1}\right]$. Identifying the logical coordinates $(i, j)$ of the B-Spline surface with the traditional notation of a finite element node, $I$, and the Cartesian product of the associated basis functions with the shape function $N_{I}(\xi, \eta)=N_{i, p}(\xi) M_{j, q}(\eta)$, the familiar finite element notation is recovered, namely:

$$
S(\xi, \eta)=\sum_{I=1}^{n \times m} N_{I}(\xi, \eta) \mathbf{x}_{I}
$$

NURBS surfaces are obtained by augmenting every point in the control mesh $\mathbf{x}_{\mathrm{I}}$ with the homogenous coordinate $w_{I}$ then dividing $S(\xi, \eta)$ by the weighting function, $w(\xi, \eta)=\sum_{I=1} N_{I}(\xi, \eta) w_{I}$, giving the final
spatial definition:

$$
S(\xi, \eta)=\frac{\sum_{I=1}^{n m} N_{I}(\xi, \eta) w_{I} \mathbf{x}_{I}}{w(\xi, \eta)}=\sum_{I=1}^{n m} \bar{N}_{I}(\xi, \eta) \mathbf{x}_{I}
$$

In equation $(6), \bar{N}_{I}(\xi, \eta)=N_{I}(\xi, \eta) w_{I} / w(\xi, \eta)$. are rational basis functions. These functions are pushed forward by the surface mapping $S(\xi, \eta)$ in equation (6) to form the approximation space for NURBSbased shell analysis. Note that $w_{I}$ are not treated as solution variables, they are data coming from the description of the NURBS surface. The concepts of control mesh, physical and parametric spaces as well as parent element are illustrated in Figure 1.

\section{Kinematics of thin shells}

In this section, the basic kinematic equations of thin shell/membrane structures are introduced. Generally, the mechanical response of membranes is naturally described by the Kirchhoff-Love shell theory [1, 6-8] augmented by the hypothesis of negligible bending stiffness. The Kirchhoff-Love shell theory accounts for membrane and bending strains which respectively arise from change of length and curvature. When the slenderness ratio of a shell structure is very large, the bending stiffness can be neglected and the equations collapse to those of a membrane. In the present study it was decided to implement an isogeometric very thin shell and assess the effect of accounting or not for the bending stiffness.

The kinematics of a thin shell, schematised in Figure 2, can be described by considering its mid-surface section as a Cosserat plane $\mathcal{A}$ and a third coordinate $\xi^{3} \in[-h / 2, h / 2]$, representing the algebraic distance to the shell mid-surface along its normal fibre where $h$ is the thickness of the shell. The mapping of the shell mid-surface is parameterised using surface curvilinear coordinates $\xi, \eta \in \mathcal{A} \subset \mathbb{R}^{2}$. The notation $\xi$, and $\eta$ for the local in-plane coordinates are abbreviated for the purpose of index notation and summation convention into $\xi^{\alpha}\left(\xi^{1}=\xi, \xi^{2}=\eta\right)$. In this paper, following standard conventions, the Einstein's summation convention is used, Latin indices range from 1 to 3 and Greek indices range from 1 to 2 . Consider a shell body whose undeformed mid-surface occupies a domain ${ }^{0} \Omega$ with boundary ${ }^{0} \Gamma$, and whose deformed mid-surface occupies a domain $\Omega$ with boundary $\Gamma$, the position vector of a material point in the geometry is given by: 


$$
\begin{gathered}
{ }^{0} \boldsymbol{\Phi}\left(\xi^{1}, \xi^{2}, \xi^{3}\right)={ }^{0} \mathbf{x}\left(\xi^{1}, \xi^{2}\right)+\xi^{30} \mathbf{t}\left(\xi^{1}, \xi^{2}\right) \\
\boldsymbol{\Phi}\left(\xi^{1}, \xi^{2}, \xi^{3}\right)=\mathbf{x}\left(\xi^{1}, \xi^{2}\right)+\xi^{3} \mathbf{t}\left(\xi^{1}, \xi^{2}\right)
\end{gathered}
$$

where ${ }^{0} \boldsymbol{\Phi}$ and $\boldsymbol{\Phi}$ correspond to the position vector of a material point associated with the convective coordinates $\left(\xi^{1}, \xi^{2}, \xi^{3}\right)$ within the shell, respectively, in its undeformed and deformed configurations. ${ }^{0} \mathbf{x}$ and $\mathbf{x}$ represent the position of a material point on the shell mid-surface $\left(\xi^{3}=0\right)$ respectively in the undeformed and deformed configurations. Similarly, ${ }^{0} \mathbf{t}$ and $\mathbf{t}$ are the unit normal vectors to the shell mid-surface for the material and spatial configurations. The mapping $\chi=\nabla \boldsymbol{\Phi} \cdot\left(\nabla^{0} \boldsymbol{\Phi}\right)^{-1}$ between these two configurations is characterised by the two-point deformation gradient:

$$
\mathbf{F}=\nabla \boldsymbol{\Phi} \cdot\left(\nabla^{0} \boldsymbol{\Phi}\right)^{-1}=\mathbf{g}_{i} \otimes{ }^{0} \mathbf{g}^{i}
$$

with

$$
\begin{aligned}
{ }^{0} \mathbf{g}_{\alpha}=\frac{\partial^{0} \mathbf{\Phi}}{\partial \xi^{\alpha}}={ }^{0} \mathbf{x}_{, \alpha}+\xi^{30} \mathbf{t}_{, \alpha} ; & { }^{0} \mathbf{g}_{3}=\frac{\partial^{0} \mathbf{\Phi}}{\partial \xi^{3}}={ }^{0} \mathbf{t} \\
\mathbf{g}_{\alpha}=\frac{\partial \boldsymbol{\Phi}}{\partial \xi^{\alpha}}=\mathbf{x}_{, \alpha}+\xi^{3} \mathbf{t}_{, \alpha} ; & \mathbf{g}_{3}=\frac{\partial \boldsymbol{\Phi}}{\partial \xi^{3}}=\mathbf{t}
\end{aligned}
$$

defining the covariant basis vectors ${ }^{0} \mathbf{g}_{i}$ and $\mathbf{g}_{i}$. The contravariant basis vectors ${ }^{0} \mathbf{g}^{i}$ and $\mathbf{g}^{i}$ follow from the relations:

$$
\mathbf{g}^{i} \cdot \mathbf{g}_{j}=\delta_{j}^{i} ; \quad{ }^{0} \mathbf{g}^{i} \cdot{ }^{0} \mathbf{g}_{j}=\delta_{j}^{i}
$$

where $\delta_{j}^{i}$ is the Kronecker delta. For subsequent developments, the co- and contravariant metric tensors are also respectively defined by equations (13) and (14):

$$
\begin{array}{ll}
{ }^{0} \mathbf{g}_{i j}={ }^{0} \mathbf{g}_{i} \cdot{ }^{0} \mathbf{g}_{j} ; & \mathbf{g}_{i j}=\mathbf{g}_{i} \cdot \mathbf{g}_{j} \\
{ }^{0} \mathbf{g}^{i j}={ }^{0} \mathbf{g}^{i} \cdot{ }^{0} \mathbf{g}^{j} ; & \mathbf{g}^{i j}=\mathbf{g}^{i} \cdot \mathbf{g}^{j}
\end{array}
$$

Using these definitions the deformation gradient can be rewritten as:

$$
\mathbf{F}=\mathbf{x}_{, \alpha} \otimes{ }^{0} \mathbf{g}^{\alpha}+\mathbf{t} \otimes{ }^{0} \mathbf{g}^{3}+\xi^{3} \mathbf{t}_{, \alpha} \otimes{ }^{0} \mathbf{g}^{\alpha}
$$

where the unit vector $\mathbf{t}$ remains always perpendicular to $\mathbf{x}_{, \alpha}$ (similarly, ${ }^{0} \mathbf{t}$ is perpendicular to ${ }^{0} \mathbf{x}_{, \alpha}$ ), due to the assumption of Kirchhoff-Love that neglects transverse shear deformations, with:

$$
\mathbf{t}=\frac{\mathbf{x}_{, 1} \times \mathbf{x}_{, 2}}{\left\|\mathbf{x}_{, 1} \times \mathbf{x}_{, 2}\right\|} ; \quad{ }^{0} \mathbf{t}=\frac{{ }^{0} \mathbf{x}_{, 1} \times{ }^{0} \mathbf{x}_{, 2}}{\left\|{ }^{0} \mathbf{x}_{, 1} \times{ }^{0} \mathbf{x}_{, 2}\right\|}
$$

If one defines the surface Jacobians ${ }^{0} \bar{j}$ and $\bar{j}$ as:

$$
{ }^{0} \bar{j}=\left\|{ }^{0} \mathbf{x}_{, 1} \times{ }^{0} \mathbf{x}_{, 2}\right\| ; \quad \bar{j}=\left\|\mathbf{x}_{, 1} \times \mathbf{x}_{, 2}\right\| \quad,
$$

the derivative of the shell director $\mathbf{t}$ follows from equation (12) as:

$$
\mathbf{t}_{, \alpha}=\frac{1}{\bar{j}}\left(\mathbf{x}_{, 1 \alpha} \times \mathbf{x}_{, 2}+\mathbf{x}_{, 1} \times \mathbf{x}_{, 2 \alpha}\right)-\frac{\mathbf{t}}{\bar{j}}\left[\left(\mathbf{x}_{, 1 \alpha} \times \mathbf{x}_{, 2}+\mathbf{x}_{, 1} \times \mathbf{x}_{, 2 \alpha}\right) \cdot \mathbf{t}\right]
$$

The Jacobian of this deformation mapping is denoted by:

$$
J=\operatorname{det}(\mathbf{F})=\frac{\operatorname{det}(\nabla \boldsymbol{\Phi})}{\operatorname{det}\left(\nabla^{0} \boldsymbol{\Phi}\right)}=\frac{\mathbf{g}_{3} \cdot\left(\mathbf{g}_{1} \times \mathbf{g}_{2}\right)}{{ }^{0} \mathbf{g}_{3} \cdot\left({ }^{0} \mathbf{g}_{1} \times{ }^{0} \mathbf{g}_{2}\right)}=\frac{j}{{ }^{0} j}
$$

The Green-Lagrange strain tensor is given by:

$$
\mathbf{E}=\frac{1}{2}\left(\mathbf{F}^{T} \mathbf{F}-\mathbf{I}\right)
$$

where $\mathbf{I}$ is the identity tensor. Ignoring the effect of a change in the shell thickness on the deformation gradient accounted by the term $\mathbf{t} \otimes{ }^{0} \mathbf{g}^{3}$ as well as the terms of order $\geq \xi^{3}$, in index notation, $\mathbf{E}$ can be written as: 


$$
E_{\alpha \beta}=\varepsilon_{\alpha \beta}+\xi^{3} \kappa_{\alpha \beta}=\frac{1}{2}\left(c_{\alpha \beta}-{ }^{0} c_{\alpha \beta}\right)+\xi^{3}\left(\omega_{\alpha \beta}-{ }^{0} \omega_{\alpha \beta}\right)
$$

where the co- and contravariant metric and curvature tensors of the surface are given by:

$$
\begin{gathered}
c_{\alpha \beta}=\mathbf{x}_{, \alpha} \cdot \mathbf{x}_{, \beta} ; \quad{ }^{0} c_{\alpha \beta}={ }^{0} \mathbf{x}_{, \alpha} \cdot{ }^{0} \mathbf{x}_{, \beta} \\
\omega_{\alpha \beta}=-\mathbf{x}_{, \alpha \beta} \cdot \mathbf{t} ; \quad{ }^{0} \omega_{\alpha \beta}=-{ }^{0} \mathbf{x}_{, \alpha \beta} \cdot{ }^{0} \mathbf{t}
\end{gathered}
$$

\subsection{Weak form of the linear momentum equation}

The weak form of the linear momentum equation is written using the principle of virtual power (see e.g., Belytschko et al. [44]): it consists in finding $\mathrm{x} \in \mathcal{V}\left(\mathrm{H}^{2}\right.$ is the space of square-integrable functions) such that:

$$
\forall \delta \mathbf{x} \in \mathcal{V}_{0} \quad \delta \Pi(\mathbf{x})=\delta \Pi_{i n t}(\mathbf{x})+\delta \Pi_{e x t}(\mathbf{x})+\delta \Pi_{k i n}(\mathbf{x})=0
$$

where:

$$
\begin{aligned}
& \mathcal{V}=\left\{\mathbf{x}(t) \mid \mathbf{x}(t) \in \mathrm{H}^{2}, \quad \mathbf{x}(t)=\overline{\mathbf{x}}(t) \text { on }{ }^{0} \Gamma^{u}\right\} \\
& \mathcal{V}_{0}=\left\{\delta \mathbf{x} \mid \delta \mathbf{x} \in \mathcal{V}, \quad \delta \mathbf{x}=0 \text { on }{ }^{0} \Gamma^{u}\right\}
\end{aligned}
$$

and

$$
\begin{gathered}
\delta \Pi_{i n t}=\int_{{ }^{0_{\Omega}}} \int_{-h / 2}^{h / 2} \boldsymbol{\tau}:\left[\delta \mathbf{x}_{, \alpha} \otimes \mathbf{g}^{\alpha}+\delta \mathbf{t} \otimes \mathbf{g}^{3}+\xi^{3} \delta \mathbf{t}_{, \alpha} \otimes \mathbf{g}^{\alpha}\right] \operatorname{det}\left(\nabla^{0} \mathbf{\Phi}\right) d \xi^{3} d^{0} \Omega \\
\delta \Pi_{e x t}=\int_{{ }^{0} \Omega} \overline{\mathbf{q}}_{0} \cdot \delta \mathbf{u} d^{0} \Omega+\int_{{ }^{0} \Gamma} \overline{\mathbf{p}}_{0} \cdot \delta \mathbf{u} d^{0} \Gamma \\
\delta \Pi_{k i n}=\int_{{ }^{0} \Omega} \rho_{0} \ddot{\mathbf{u}} \cdot \delta \mathbf{u} d^{0} \Omega
\end{gathered}
$$

where $\mathbf{u}$ is the deformation vector, $\delta$ denotes variations, $\Pi_{\text {ert }}$ is the external energy, $\Pi_{\text {int }}$ designates the internal energy, and $\Pi_{k i n}$ the kinetic energy [44]. $\boldsymbol{\tau}$ is the Kirchhoff stress tensor, $\rho_{0}$ is the density, $\overline{\mathbf{q}}_{0}$ is the external body load per unit area and $\overline{\mathbf{p}}_{0}$ is the forces per unit length of the boundary of the undeformed mid-surface; superposed dots denote material time derivatives. Following Simo and Fox [8] and Simo et al. [7], the integration through the thickness of the Kirchhoff stress tensor $\boldsymbol{\tau}$ leads to the definitions:

$$
\begin{aligned}
\mathbf{n}^{\alpha} & =\frac{1}{\bar{j}} \int_{-h / 2}^{h / 2} \boldsymbol{\tau} \mathbf{g}^{\alpha} \operatorname{det}\left(\nabla^{0} \mathbf{\Phi}\right) d \xi^{3} \\
\tilde{\mathbf{m}}^{\alpha} & =\frac{1}{\bar{j}} \int_{-h / 2}^{h / 2} \xi^{3} \boldsymbol{\tau} \mathbf{g}^{\alpha} \operatorname{det}\left(\nabla^{0} \boldsymbol{\Phi}\right) d \xi^{3} \\
\mathbf{l} & =\frac{1}{\bar{j}} \int_{-h / 2}^{h / 2} \boldsymbol{\tau} \mathbf{g}^{3} \operatorname{det}\left(\nabla^{0} \boldsymbol{\Phi}\right) d \xi^{3}
\end{aligned}
$$

Substituting equations (29), (30) and (31) into equation (24) yields:

$$
\delta \Pi_{\text {int }}=\int_{{ }_{0} \Omega}\left(\mathbf{n}^{\alpha} \cdot \delta \mathbf{x}_{, \alpha}+\tilde{\mathbf{m}}^{\alpha} \cdot \delta \mathbf{t}_{, \alpha}+\mathbf{l} \cdot \delta \mathbf{t}\right) d^{0} \Omega
$$

Since the Kirchhoff-Love assumption requires that $\mathbf{t}_{\alpha} \cdot \mathbf{t}=0$, the deformation gradient (equation (9)) has null components along $\alpha 3$ and $3 \alpha$ in the convected basis. Therefore, for elasticity, the Kirchhoff stress tensor components read:

$$
\tau^{i 3}=\tau^{i 3}=0
$$

and the resultant vectors can be rewritten as:

$$
\begin{aligned}
\mathbf{n}^{\alpha} & =\frac{1}{\bar{j}} \int_{-h / 2}^{h / 2} \tau^{\alpha \beta} \mathbf{g}_{\beta} \operatorname{det}\left(\nabla^{0} \mathbf{\Phi}\right) d \xi^{3} \\
\tilde{\mathbf{m}}^{\alpha} & =\frac{1}{\bar{j}} \int_{-h / 2}^{h / 2} \xi^{3} \tau^{\alpha \beta} \mathbf{g}_{\beta} \operatorname{det}\left(\nabla^{0} \boldsymbol{\Phi}\right) d \xi^{3}
\end{aligned}
$$




$$
\mathbf{l}=\frac{1}{\bar{j}} \int_{-h / 2}^{h / 2} \tau^{i 3} \mathbf{g}_{i} \operatorname{det}\left(\nabla^{0} \mathbf{\Phi}\right) d \xi^{3}=0
$$

where $\mathbf{n}^{\alpha}$ corresponds to the resultant membrane stress vectors, $\tilde{\mathbf{m}}^{\alpha}$ the resultant bending stress (torque) vectors and 1 the resultant across-the-thickness stress vector. To demonstrate the stability of the linearised form, new definitions are introduced, see $[7,8]$ for details. Since $\mathbf{t}_{{ }_{\alpha}} \cdot \mathbf{t}=0$, the gradient of the unit normal vector can be expressed in the mid-surface convected basis as:

$$
\mathbf{t}_{, \alpha}=\lambda_{\alpha}^{\mu} \mathbf{x}_{, \mu}+\lambda_{\alpha}^{3} \mathbf{t}
$$

Similarly, in order to define the stress components, the resultant stress vectors are decomposed in this midsurface convected basis as:

$$
\begin{gathered}
\mathbf{n}^{\alpha}=n^{\alpha \beta} \mathbf{x}_{\beta \beta}+q^{\alpha} \mathbf{t} \\
\tilde{\mathbf{m}}^{\alpha}=\tilde{m}^{\alpha \beta} \mathbf{x}_{, \beta}+\tilde{m}^{3 \alpha} \mathbf{t} \\
\mathbf{l}=l^{\alpha} \mathbf{x}_{, \alpha}+l^{3} \mathbf{t}
\end{gathered}
$$

Since the Cauchy stress tensor is symmetric, the following expression holds:

$$
\mathbf{n}^{\alpha} \times \mathbf{x}_{, \alpha}+\tilde{\mathbf{m}}^{\alpha} \times \mathbf{t}_{, \alpha}+\mathbf{l} \times \mathbf{t}=\mathbf{0}
$$

and can be satisfied by enforcing the symmetry of the effective membrane stress resultant tensor, i.e.:

$$
\tilde{n}^{\alpha \beta} \mathbf{x}_{, \alpha} \otimes \mathbf{x}_{, \beta}=\mathbf{n}^{\alpha} \otimes \mathbf{x}_{, \alpha}+\mathbf{l} \otimes \mathbf{t}-\mathbf{t}_{, \alpha} \otimes \tilde{\mathbf{m}}^{\alpha}=\tilde{n}^{\alpha \beta} \mathbf{x}_{, \beta} \otimes \mathbf{x}_{, \alpha}
$$

From equations (38), (39), (40) and (42), the effective membrane stress resultant $\tilde{n}^{i j}$ can be obtained by:

$$
\begin{aligned}
& \tilde{n}^{\alpha \beta}=n^{\alpha \beta}-\lambda_{\mu}^{\beta} \tilde{m}^{\alpha \mu}=\tilde{n}^{\beta \alpha} \\
& \tilde{n}^{3 \alpha}=q^{\alpha}-\lambda_{\mu}^{3} \tilde{m}^{\alpha \mu}=\tilde{q}^{\alpha} \\
& \tilde{n}^{\alpha 3}=l^{\alpha}-\lambda_{\mu}^{\alpha} \tilde{m}^{3 \mu}=\tilde{l}^{\alpha}=\tilde{q}^{\alpha}
\end{aligned}
$$

and by:

$$
\tilde{n}^{33}=\tilde{l}^{3}-\lambda_{\mu}^{3} \tilde{m}^{3 \mu}=\tilde{l}^{3}
$$

which are respectively the membrane effective stresses, effective transverse shear, effective symmetric shear and effective across-the-thickness normal stress. Since Kirchhoff-Love shells are assumed to be in a state of plane stress [1], the following expression holds:

$$
\tilde{n}^{33}=\tilde{l}^{\alpha}=\tilde{q}^{\alpha}=\tilde{n}^{3 \alpha}=\tilde{l}^{3}=0
$$

For a linear elastic material, the resultant effective membrane stress and bending stress can be expressed by $[7$, 8]:

with

$$
\begin{aligned}
& \tilde{n}^{\alpha \beta}=h \mathcal{H}^{\alpha \beta \gamma \delta} \varepsilon_{\alpha \beta} \\
& \tilde{m}^{\alpha \beta}=\frac{h^{3}}{12} \mathcal{H}^{\alpha \beta \gamma \delta} \kappa_{\alpha \beta}
\end{aligned}
$$

$$
\mathcal{H}^{\alpha \beta \gamma \delta}=\frac{E}{1-v^{2}}\left[v^{0} \mathbf{x}^{\alpha} \cdot{ }^{0} \mathbf{x}^{, \beta}{ }^{0} \mathbf{x}^{, \gamma} \cdot{ }^{0} \mathbf{x}^{, \delta}+\frac{1}{2}(1-v)^{0} \mathbf{x}^{, \alpha} \cdot{ }^{0} \mathbf{x}^{, \gamma}{ }^{0} \mathbf{x}^{, \delta} \cdot{ }^{0} \mathbf{x}^{, \beta}+\frac{1}{2}(1-v)^{0} \mathbf{x}^{, \alpha} \cdot{ }^{0} \mathbf{x}^{, \delta}{ }^{0} \mathbf{x}^{, \gamma} \cdot{ }^{0} \mathbf{x}^{, \beta}\right]
$$

$\mathcal{H}$ is the constitutive tensor of the isotropic St. Venant-Kirchhoff material under the plane stress condition, in which $E$ is the Young's modulus and $v$ is the Poisson's ratio.

Substituting equations (38)-(46) into the internal energy of equation (32) and linearising the non-linear model, then: 


$$
\begin{aligned}
\delta \Pi_{i n t} & =\int_{{ }^{\Omega} \Omega}(\mathbf{n}^{\alpha} \cdot \delta \mathbf{x}_{, \alpha}+\tilde{\mathbf{m}}^{\alpha} \cdot \delta \mathbf{t}_{, \alpha}+\overbrace{\mathbf{l} \cdot \delta \mathbf{t}}^{0}) d^{0} \Omega \\
& =\int_{{ }_{0} \Omega}(\overbrace{\tilde{n}^{\alpha \beta 0} \mathbf{x}_{, \beta} \cdot \delta \mathbf{u}_{, \alpha}+\tilde{m}^{\alpha \beta} \mathbf{t}_{, \beta} \cdot \delta \mathbf{u}_{, \alpha}}^{\mathbf{n}^{\alpha} \cdot \delta \Phi_{\alpha}}+\overbrace{\tilde{m}^{\alpha \beta 0} \mathbf{x}_{, \beta} \cdot \delta \mathbf{t}_{, \alpha}}^{\tilde{\mathbf{m}}^{\alpha} \cdot \delta \mathbf{t}_{, \alpha}}) d^{0} \Omega \\
& =\int_{{ }_{0}}\left(\tilde{n}^{\alpha \beta} \cdot \delta \varepsilon_{\alpha \beta}+\tilde{m}^{\alpha \beta} \cdot \delta \kappa_{\alpha \beta}\right) d^{0} \Omega
\end{aligned}
$$

Using Voigt's notation one can write:

$$
\tilde{\mathbf{n}}=\left(\begin{array}{l}
\tilde{n}^{11} \\
\tilde{n}^{22} \\
\tilde{n}^{12}
\end{array}\right)=h \mathbf{H}\left(\begin{array}{c}
\overbrace{\varepsilon^{11}}^{\varepsilon} \\
\varepsilon^{22} \\
\varepsilon^{12}
\end{array}\right) ; \quad \tilde{\mathbf{m}}=\left(\begin{array}{c}
\tilde{m}^{11} \\
\tilde{m}^{22} \\
\tilde{m}^{12}
\end{array}\right)=\frac{h^{3}}{12} \mathbf{H} \overbrace{\left(\begin{array}{c}
\kappa^{11} \\
\kappa^{22} \\
\kappa^{12}
\end{array}\right)}^{\kappa}
$$

where:

$$
\mathbf{H}=\frac{E}{1-v^{2}}\left[\begin{array}{ccc}
a_{0}^{11} a_{0}^{11} & v a_{0}^{11} a_{0}^{22}+(1-v) a_{0}^{12} a_{0}^{12} & a_{0}^{11} a_{0}^{12} \\
v a_{0}^{11} a_{0}^{22}+(1-v) a_{0}^{12} a_{0}^{12} & a_{0}^{22} a_{0}^{22} & a_{0}^{22} a_{0}^{12} \\
a_{0}^{11} a_{0}^{12} & a_{0}^{22} a_{0}^{12} & \frac{1-v}{2} a_{0}^{11} a_{0}^{22}+\frac{1+v}{2} a_{0}^{12} a_{0}^{12}
\end{array}\right]
$$

in which. $a_{0}^{\alpha \beta}={ }^{0} \mathbf{x}^{\alpha} \cdot{ }^{0} \mathbf{x}^{\beta}$. Correspondingly, equation (48) can be rewritten in a compact form as:

$$
\delta \Pi_{i n t}=\int_{{ }^{0} \Omega}(\tilde{\mathbf{n}} \cdot \delta \varepsilon+\tilde{\mathbf{m}} \cdot \delta \boldsymbol{\kappa}) d^{0} \Omega
$$

\subsection{Non-linear elastic constitutive law}

In addition to the linear elastic material for which constitutive equations were established in the previous section using an Eulerian viewpoint, the present IGA framework includes a non-linear elastic (hyperelastic) constitutive law, for which, however, the second Piola-Kirchhoff membrane stress $\mathbf{S}$ are alternatively utilised for this purpose.

As an example of hyperelastic behaviour, herein, we consider an Ogden material [48] whose behaviour is characterised by a strain energy density $W$ that is an isotropic function of the principal stretches $\lambda_{i}$, and is written as

$$
W\left(\lambda_{i}\right)=\sum_{r} \frac{\mu_{r}}{\alpha_{r}}\left(\lambda_{1}^{\alpha_{r}}+\lambda_{2}^{\alpha_{r}}+\lambda_{3}^{\alpha_{r}}-3\right)
$$

where $\mu_{r}$ and $\alpha_{r}$ are constants. The exponents $\alpha_{r}$ may take any non-zero real value. The summation on $r$ extends over as many terms as are necessary to characterise a particular material. Using the incompressibility constraint $\lambda_{3}=\left(\lambda_{1} \lambda_{2}\right)^{-1}$ the strain energy can be expressed as a function of the independent stretches $\lambda_{1}$ and $\lambda_{2}$

$$
W\left(\lambda_{i}\right)=\sum_{r} \frac{\mu_{r}}{\alpha_{r}}\left(\lambda_{1}^{\alpha_{r}}+\lambda_{2}^{\alpha_{r}}+\left(\lambda_{1} \lambda_{2}\right)^{-\alpha_{r}}-3\right)
$$

The second Piola-Kirchhoff stress tensor $\mathbf{S}$ follows from differentiation of $W$ with respect to the work conjugate Green-Lagrange strain tensor. Using the chain rule the components of $\mathbf{S}$ are given by

$$
S^{\alpha \beta}=\frac{\partial W}{\partial E_{\alpha \beta}}=\frac{\partial W}{\partial \lambda_{1}} \frac{\partial \lambda_{1}}{\partial E_{\alpha \beta}}+\frac{\partial W}{\partial \lambda_{2}} \frac{\partial \lambda_{2}}{\partial E_{\alpha \beta}}
$$

Owing to the plane stress assumption the stresses $S^{\alpha 3}$ are vanishing. Next, we define the principal values $S_{r}$, of the Second Piola-Kirchhoff stress tensor:

$$
S_{\gamma}=\lambda_{\gamma}^{-1} \frac{\partial W}{\partial \lambda_{\gamma}}=\lambda_{\gamma}^{-2} \sum_{r} \mu_{r}\left(\lambda_{\gamma}^{\alpha_{r}}-\left(\lambda_{1} \lambda_{2}\right)^{-\alpha_{r}}\right)
$$


The Kirchhoff stress can be obtained by standard push-forward operation on $\mathbf{S}$ [49]:

$$
\tau_{\gamma}=\lambda_{\gamma}^{2} S_{\gamma}=\lambda_{\gamma} \frac{\partial W}{\partial \lambda_{\gamma}}=\sum_{r} \mu_{r}\left(\lambda_{\gamma}^{\alpha_{r}}-\left(\lambda_{1} \lambda_{2}\right)^{-\alpha_{r}}\right)
$$

\section{Implementation}

\subsection{Dynamic relaxation method}

In order to obtain solutions of the shell/membrane structures at steady state, a dynamic relaxation method $[17,46]$ is employed and combined with a central difference explicit time integration algorithm [46]. Firstly, one can formulate the discrete equations of motion of a structure as follows:

$$
\mathbf{M u ̈}=\mathbf{f}^{e x t}-\mathbf{f}^{i n t}-\mathbf{f}^{\text {damping }}
$$

subject to:

$$
g_{i b c}\left({ }^{t} \dot{\mathbf{u}}\right)=0, i b c=1 \text { to } n_{b c}
$$

where $\ddot{\mathbf{u}}$ and $\dot{\mathbf{u}}$ are the acceleration and velocity vectors in the global Cartesian coordinates, $\mathbf{M}$ is the lumped mass matrix. $g_{i b c}\left({ }^{t} \dot{\mathbf{u}}\right)=0$ is the $i$-th velocity boundary condition at time $t$, and $n_{b c}$ is the number of boundary conditions. $\mathbf{f}^{\text {int }}, \mathbf{f}^{\text {ext }}$ and $\mathbf{f}^{\text {damping }}$ are respectively the internal, external and damping force vectors. In the present research, the following form is adopted for $\mathbf{f}^{\text {damping }}[17]$.

$$
\mathbf{f}^{\text {damping }}=\mu \mathbf{M} \dot{\mathbf{u}}
$$

where $\mu$ is a positive damping coefficient. It is noteworthy that, here, the damping is not a physical parameter (generally associated with material viscosity and strain rate effects). The damping force in equation (59) is used to artificially dissipate the kinetic energy so that a steady state solution of the membrane structure can be obtained relatively quickly whilst minimising the potential influence of the transient response (inertial forces) on the final sought quasi-static solution. Hence, the selection of a positive damping coefficient $\mu$ in the present research is not required, and the empirical value of the damping coefficient only affects the total number of time steps to reach the steady state.

\subsection{Discretisation and evaluation of the residual and mass matrices}

The assumed large deformation kinematics employs the Kirchhoff-Love theory to describe the mechanical behaviour of shells. The displacement fields are interpolated from the displacements of control points only, and no rotational degrees of freedom are used at control points. This leads to a particularly simple representation that can be expressed by:

$$
{ }^{0} \mathbf{x}_{h}=\sum_{I=1}^{N_{n}} \bar{N}_{I}^{0} \mathbf{x}_{I} ; \quad \mathbf{x}_{h}=\sum_{I=1}^{N_{n}} \bar{N}_{I} \mathbf{x}_{I}
$$

for the undeformed and deformed mid-surfaces of the shell, respectively. In equation (60), ${ }^{0} \mathbf{x}_{I}$ and $\mathbf{x}_{I}$ are the coordinates of control points with total number of $N_{n}=n \times m$, and $\bar{N}_{I}$ are the NURBS-based shape functions, as defined in equation (6). $\bar{N}$ is termed as $N$ in the following sections for convenience. ${ }^{0} \mathbf{x}_{h}$ and $\mathbf{x}_{h}$ are the coordinates of the undeformed and deformed mid-surfaces of the shell.

\section{Remark about continuity:}

In theory, the $C^{k}(k \geq 1)$ continuity of NURBS shape functions with order $p \geq 2$ is ensured. The displacement field is guaranteed to be $\mathrm{H}^{2}$, i.e. to be square-integrable and to have first and second squareintegrable derivatives. In consequence, the interpolated displacement fields have a finite Kirchoff-Love energy. The proposed interpolation scheme is of continuity strictly higher than $C^{1}$ and therefore meets the convergence requirements of the displacement method, thereby side-stepping the difficulties associated with the use of $C^{0}$ methods in the limit of vanishing shell thickness [20]. Introduction of the discretisation scheme (equation (60)) into the weak form of equation (24) yields a semi-discrete system of equations of the form: 


$$
\mathbf{M}_{h} \ddot{\mathbf{u}}+\mu \mathbf{M}_{h} \dot{\mathbf{u}}+\mathbf{f}_{h}^{\text {int }}=\mathbf{f}_{h}^{e x t}
$$

where the internal forces are expressed as:

$$
\mathbf{f}_{h}^{i n t}=\int_{{ }_{0} \Omega}\left(\mathbf{B}_{m}^{T} \tilde{\mathbf{n}}+\mathbf{B}_{b}^{T} \tilde{\mathbf{m}}\right) d^{0} \Omega
$$

As in the standard finite element method, the global internal force array is the sum of NURBS-based element contributions, each of which entails the computation of an integral extended to the domain of one element, as:

$$
\mathbf{f}_{h}^{i n t}=\int_{{ }_{0}}\left(\mathbf{B}_{m}^{T} \tilde{\mathbf{n}}+\mathbf{B}_{b}^{T} \tilde{\mathbf{m}}\right) d^{0} \Omega=\sum_{L=1}^{N_{e}} \mathbf{f}_{h L}^{i n t}=\sum_{L=1}^{N_{e}} \int_{{ }_{L} \Omega}\left(\mathbf{B}_{m}^{T} \tilde{\mathbf{n}}+\mathbf{B}_{b}^{T} \tilde{\mathbf{m}}\right) d_{L}^{0} \Omega
$$

where $N_{e}$ is the number of NURBS based elements in the problem domain. $\mathbf{B}_{m}$ and $\mathbf{B}_{b}$ are respectively the membrane and bending-stain matrices that take the form:

$$
\left[\mathbf{B}_{m}\right]=\prod_{I=1}^{n_{k}^{e}} \mathbf{B}_{m}^{I} ; \quad\left[\mathbf{B}_{b}\right]=\prod_{I=1}^{n_{k}^{e}} \mathbf{B}_{b}^{I}
$$

where $n_{k}^{e}$ is the set of control points associated with the element ${ }_{L}^{0} \Omega$. With the displacement vector expressed by $\mathbf{u}=\mathbf{x}-{ }^{0} \mathbf{x}$, the linear membrane and bending strain measures can be derived from the kinematic variables in equations. (22) and (23) as:

$$
\begin{aligned}
\varepsilon_{\alpha \beta} & =\frac{1}{2}\left({ }^{0} \mathbf{x}_{, \alpha} \cdot \mathbf{u}_{, \beta}+{ }^{0} \mathbf{x}_{, \beta} \cdot \mathbf{u}_{, \alpha}\right) \\
\kappa_{\alpha \beta} & =\frac{1}{2}\left({ }^{0} \mathbf{x}_{, \alpha} \cdot \Delta \mathbf{t}_{, \beta}+{ }^{0} \mathbf{x}_{, \beta} \cdot \Delta \mathbf{t}_{, \alpha}+\mathbf{u}_{, \alpha} \cdot{ }^{0} \mathbf{t}_{, \beta}+\mathbf{u}_{, \beta} \cdot{ }^{0} \mathbf{t}_{, \alpha}\right)
\end{aligned}
$$

where by retaining only terms linear in $\mathbf{u}$, and by invoking the condition $\mathbf{t}_{, \alpha} \cdot \mathbf{t}=0$, the derivative of the normal vector increment of $\Delta \mathbf{t}=\mathbf{t}-{ }^{0} \mathbf{t}$ is obtained as:

$$
\Delta \mathbf{t}_{, \alpha}=\frac{1}{{ }^{0} \bar{j}}\left(\mathbf{u}_{, 1 \alpha} \times{ }^{0} \mathbf{x}_{, 2}+\mathbf{u}_{, 1} \times{ }^{0} \mathbf{x}_{, 2 \alpha}+{ }^{0} \mathbf{x}_{, 1 \alpha} \times \mathbf{u}_{, 2}+{ }^{0} \mathbf{x}_{, 1} \times \mathbf{u}_{, 2 \alpha}\right)
$$

Substituting equation (62) into equation (61), the membrane and bending strain matrices associated with node $I, \mathbf{B}_{m}^{I}$ and $\mathbf{B}_{b}^{I}$, can be computed as:

and

$$
\begin{gathered}
\mathbf{B}_{m}^{I}=\left[\begin{array}{ccc}
N_{, 1}^{I 0} \mathbf{x}_{, 1} \cdot \mathbf{e}_{1} & N_{, 1}^{I 0} \mathbf{x}_{, 1} \cdot \mathbf{e}_{2} & N_{, 1}^{I 0} \mathbf{x}_{, 1} \cdot \mathbf{e}_{3} \\
N_{, 2}^{I 0} \mathbf{x}_{, 2} \cdot \mathbf{e}_{1} & N_{, 2}^{I 0} \mathbf{x}_{, 2} \cdot \mathbf{e}_{2} & N_{, 2}^{I 0} \mathbf{x}_{, 2} \cdot \mathbf{e}_{3} \\
\left(N_{, 1}^{I 0} \mathbf{x}_{, 2}+N_{, 2}^{I 0} \mathbf{x}_{, 1}\right) \cdot \mathbf{e}_{1} & \left(N_{, 1}^{I 0} \mathbf{x}_{, 2}+N_{, 2}^{I 0} \mathbf{x}_{, 1}\right) \cdot \mathbf{e}_{2} & \left(N_{, 1}^{I 0} \mathbf{x}_{, 2}+N_{, 2}^{I 0} \mathbf{x}_{, 1}\right) \cdot \mathbf{e}_{3}
\end{array}\right] \\
\mathbf{B}_{b}^{I}=\left[\begin{array}{lll}
\mathbf{B}_{11 b}^{I} \cdot \mathbf{e}_{1} & \mathbf{B}_{11 b}^{I} \cdot \mathbf{e}_{2} & \mathbf{B}_{111}^{I} \cdot \mathbf{e}_{3} \\
\mathbf{B}_{22 b}^{I} \cdot \mathbf{e}_{1} & \mathbf{B}_{22 b}^{I} \cdot \mathbf{e}_{2} & \mathbf{B}_{22}^{I} \cdot \mathbf{e}_{3} \\
\mathbf{B}_{12 b}^{I} \cdot \mathbf{e}_{1} & \mathbf{B}_{12 b}^{I} \cdot \mathbf{e}_{2} & \mathbf{B}_{12 b}^{I} \cdot \mathbf{e}_{3}
\end{array}\right]
\end{gathered}
$$

In the above expressions, $\mathbf{e}_{1}, \mathbf{e}_{2}$ and $\mathbf{e}_{3}$ are the global basis vectors of an orthonormal coordinate frame, and:

$$
\mathbf{B}_{\alpha \beta b}^{I}=-N_{, \alpha \beta}^{I}{ }^{0} \mathbf{t}+\frac{1}{{ }^{0} \bar{j}}\left[N_{, 1}^{I 0} \mathbf{x}_{, \alpha \beta} \times{ }^{0} \mathbf{x}_{, 2}+N_{, 2}^{I 0} \mathbf{x}_{, \alpha \beta} \times{ }^{0} \mathbf{x}_{, 1}+{ }^{0} \mathbf{t} \cdot{ }^{0} \mathbf{x}_{, \alpha \beta}\left(N_{, 1}^{I 0} \mathbf{x}_{, 2} \times{ }^{0} \mathbf{t}+N_{, 2}^{I 0} \mathbf{x}_{, 1} \times{ }^{0} \mathbf{t}\right)\right]
$$

Using Gauss integration in the element domain ${ }_{L}^{0} \Omega$, we have:

$$
\mathbf{f}_{h}^{\text {int }}==\sum_{L=1}^{N_{e}} \sum_{G=1}^{N_{\text {Ganus }}}\left\{\left(\mathbf{B}_{m}^{T} \tilde{\mathbf{n}}+\mathbf{B}_{b}^{T} \tilde{\mathbf{m}}\right)^{0} \bar{j}\right\}_{\left(\xi_{G}^{1}, \xi_{G}^{2}\right)} w_{G}
$$

where $N_{\text {Gauss }}$ is the number of Gauss points used in each segment, $\left(\xi_{G}^{1}, \xi_{G}^{2}\right)$ are the surface coordinates of the Gauss points of the element and $w_{G}$ is the corresponding Gaussian quadrature weight. We additionally compute the effective stress resultants $\tilde{\mathbf{n}}$ and $\tilde{\mathbf{m}}$ using equation (49). The mass matrix follows likewise as:

$$
\mathbf{M}_{h}^{I J}=\sum_{L=1}^{N_{e}} \sum_{G=1}^{N_{G \text { Gusses }}}\left\{\left(\rho_{0} h N^{I} N^{J}\right)^{0} \bar{j}\right\}_{\left(\xi_{G}^{1}, \xi_{G}^{2}\right)} w_{G}
$$




\subsection{Numerical procedure}

The detailed procedure to solve the equations of motion using the dynamic relaxation method (see section 4.1) based on the central difference explicit time integration algorithm with constant time step is given as follows:

1. Discretise and compute the mass matrix $\mathbf{M}$

2. Loop over all the NURBS based elements ${ }_{L}^{0} \Omega\left(L=1\right.$ to $\left.N_{\text {el }}\right)$

3. Set initial conditions, ${ }^{0} \dot{\mathbf{u}}_{I}\left(I=1 . . N_{n}\right),{ }^{0} \mathbf{u}_{I}=\mathbf{0}\left(I=1 . . N_{n}\right), t(\tau)=0$, and the counter $\tau=0$

4. Call subroutine Compute_Internal_Forces to compute the internal force vector ${ }^{t(\tau)} \mathbf{f}^{\text {int }}$ using the displacements of control points

5. Compute force ${ }^{t(\tau)} \mathbf{f}={ }^{t(\tau)} \mathbf{f}^{e x t}-{ }^{t(\tau)} \mathbf{f}^{\text {int }}-{ }^{t(\tau)} \mathbf{f}^{\text {damping }}$

6. Compute accelerations of control points ${ }^{t(\tau)} \mathbf{a}=\mathbf{M}^{-1} \cdot{ }^{t(\tau)} \mathbf{f}$

7. Time update $t(\tau+1)=t(\tau)+\Delta t, t(\tau+1 / 2)=t(\tau)+\Delta t / 2$

8. Partial update velocities, ${ }^{t(\tau+1 / 2)} \dot{\mathbf{u}}={ }^{t(\tau)} \dot{\mathbf{u}}+\Delta t \cdot{ }^{t(\tau)} \ddot{\mathbf{u}} / 2$

9. Apply velocity boundary conditions $g_{i b c}\left({ }^{t(\tau+1 / 2)} \dot{\mathbf{u}}\right)=0, i b c=1$ to $n_{b c}$

10. Update displacement of control points ${ }^{t(\tau+1)} \mathbf{u}={ }^{t(\tau)} \mathbf{u}+\Delta t^{t(\tau+1 / 2)} \dot{\mathbf{u}}$

11. Compute force ${ }^{t(\tau+1)} \mathbf{f}={ }^{t(\tau+1)} \mathbf{f}^{\text {ext }}-{ }^{t(\tau+1)} \mathbf{f}^{\text {int }}-{ }^{t(\tau+1)} \mathbf{f}^{\text {damping }}$

12. Compute accelerations of control points ${ }^{t(\tau+1)} \mathbf{a}=\mathbf{M}^{-1} \cdot{ }^{t(\tau+1)} \mathbf{f}$

13. Update velocities of control points, ${ }^{t(\tau+1)} \dot{\mathbf{u}}={ }^{t(\tau+1 / 2)} \dot{\mathbf{u}}+\Delta t \cdot{ }^{t(\tau+1)} \ddot{\mathbf{u}} / 2$

14. Check convergence: if conditions (68)-(70) are satisfied, the steady state solution is obtained for the load level $\mathfrak{F}$. If NOT, update the counter $\tau \leftarrow \tau+1$ and go to Step (1).

$$
\begin{gathered}
{ }^{t} \mathbf{f}^{e x t}=\mathfrak{F} \\
\left\|{ }^{t(\tau+1)} \mathbf{f}^{e x t}-{ }^{t(\tau+1)} \mathbf{f}^{i n t}\right\|_{L 2} /\|\|^{t(\tau+1)} \mathbf{f}^{e x t} \|_{L 2}<\varepsilon_{F} \\
\left\|{ }^{t(\tau+1)} \mathbf{u}-{ }^{t(\tau)} \mathbf{u}\right\|_{L 2} /\|\|^{t(\tau)} \mathbf{u} \|_{L 2}<\varepsilon_{U}
\end{gathered}
$$

Subroutine Compute_Internal_Forces:

Loop over all the NURBS elements ${ }^{0} \Omega_{L}^{e}\left(L=1\right.$ to $\left.N_{e l}\right)$

Loop over all Gaussian points in one element

(S.1) Get the current coordinates $\mathbf{x}_{I}={ }^{0} \mathbf{x}_{I}+\mathbf{u}_{I}$ for all control points

(S.2) Compute the membrane and bending strains $\boldsymbol{\varepsilon}\left(\mathbf{x}_{G}\right)$ and $\mathbf{\kappa}\left(\mathbf{x}_{G}\right)$ using equation (21)

(S.3) Compute the effective stress resultants $\tilde{\mathbf{n}}$ and $\tilde{\mathbf{m}}$ using equation (49)

(S.4) Compute the membrane and bending-stain matrices $\mathbf{B}_{m}$ and $\mathbf{B}_{b}$ using equations (67)-(69)

(S.5) Compute $\mathbf{f}_{h L}^{\text {int }}$ and assemble to $\mathbf{f}_{h}^{\text {int }}$.

The admissible errors $\varepsilon_{F}$ and $\varepsilon_{U}$ in equations (73) and (74) are set to be very small value $\left(10^{-7}\right)$ in the present research so as to get steady state solutions with minimal influence of the transient responses. 


\subsection{Critical time step}

It should be noted that the explicit time integration algorithm is conditionally stable, which requires the time step $\Delta t$ not to exceed a critical value $\Delta t_{\text {crit }}$ according to the Courant-Friedrichs-Lewy condition [44]. A stable time step for a rate-independent material can be determined as:

$$
\Delta t=\alpha \Delta t_{\text {crit }}, \Delta t_{\text {crit }}=\frac{2 \pi}{\omega_{\max }} \leq \min _{e}\left(\frac{\ell_{e}}{c_{e}}\right)
$$

where, $\ell_{e}$ is the characteristic length of the element $e, c_{e}$ is the wave speed in element $e$, and $\alpha$ is a reduction factor that accounts for the destabilising effects of non-linearities. $\alpha$ can be typically chosen as $\alpha \in[0.8,0.98]$. In this work, the constant sound speed for plane stress problems is evaluated using:

$$
c=\sqrt{\frac{E}{\left(1-v^{2}\right) \rho}}
$$

Furthermore, the present research mainly focuses on the accuracy in terms of the steady state solutions solved using a dynamic relaxation scheme. At the steady state, the kinetic energy of the structure is dissipated and the choice of the density has little influence on the steady state solutions in terms of displacement and strain energy for path-independent and rate-independent hyperelastic materials. A faster convergence procedure can therefore be obtained by increasing the density which has the effect of enabling the use of larger critical time step $\Delta t_{\text {crit }}$.

\section{Numerical examples}

The NURBS-based isogeometric thin shell described in the previous section was tested through a series of benchmark examples.

\subsection{Example 1- Square membrane under static pressure}

Our first verification test concerns the simple case of a square membrane made of DuPont ${ }^{\mathrm{TM}}$ Tyvek $\mathbb{R}$ (DuPont, Wilmington, DE, USA) home wrap subjected to a uniform static pressure, with four corners constrained in $x, y$ and $z$ directions by regularly spaced stables or caps. The dimension of the membrane are the following: length and width are $406 \mathrm{~mm}$ while the thickness is $t=0.16 \mathrm{~mm}$. The material properties of the membrane are: Young's modulus $E=875 \mathrm{MPa}$, Poisson's ratio $v=0.24$ and density $\rho=1 \times 10^{3} \mathrm{~kg} / \mathrm{m}^{3}$. The damping coefficient is $\mu=5 \mathrm{~N} \cdot \mathrm{s} /(\mathrm{kg} \cdot \mathrm{m})$. The membrane is assumed to be at rest in the undeformed configuration. Four different static pressure values were considered: $p_{\text {int }}=25 \mathrm{~Pa}$, $50 \mathrm{~Pa}, 100 \mathrm{~Pa}, 150 \mathrm{~Pa}$. For each of the four analyses both the resulting maximum deflection $H$ and the deflections along the diagonal and the edge are investigated. The geometry, loading and boundary conditions are depicted in Figure 3. An appealing feature of the problem under consideration is that it is directly comparable to a reference solution that originates from the experimental work described in Shi et al. [50]. Moreover, this example was studied previously using other approaches such as a theoretical model with linear assumptions [51], and finite element analyses using the commercial code ABAQUS (Simulia, Dassault Systèmes, Providence, RI, USA) [52]. The corresponding results are thus given for comparison. In addition to the experimental solutions, other reference solutions are calculated using 3-noded thin shell elements (type S3) available in the element library of ABAQUS. In this instance a very fine mesh consisting of 75209 nodes and 149784 elements is used. The numerical solution obtained from ABAQUS is herein referred as the "reference solution" while the experimental one is referred as the "experimental solution". These two sets of results provide a convenient basis for the assessment of the accuracy and convergence properties of the present isogeometric analysis. Using a slightly abusive terminology, finite element models based on Lagrange polynomial shape functions are herein referred to "FEM" models.

In the present numerical scheme, the NURBS IGA model with both zero bending stiffness (NURBS IGA/Membrane) and non-zero bending stiffness (NURBS IGA/Shell) are included. A constant sub-critical time step $\Delta t=2 \times 10^{-3} \mathrm{~s}$ is employed. Meshes of quadratic, cubic and quartic $(p=2,3$, and 4$)$ NURBS surface elements with different levels of $k$-type refinement $(3 \times 3,6 \times 6,12 \times 12$ and $24 \times 24)[18]$ are considered in the computations. These four levels of refinement are depicted in Figure 4. Note that $p+1$ Gauss points in each in-plane direction (for each knot interval) are used, unless specified otherwise. 


\subsubsection{Effect of the bending stiffness}

Table 1-3 presents the results of the study of the influence of the bending stiffness on the following three response variables:

(1) the maximum deflection of the membrane at the steady state;

(2) the deflections at points $\mathrm{D}_{1}, \mathrm{D}_{2}, \ldots, \mathrm{D}_{8}$ along the diagonal; and

(3) the deflections at points $\mathrm{E}_{1}, \mathrm{E}_{2}, \ldots, \mathrm{E}_{8}$ along the edge (see Figure 3).

In this study, quadratic $(p=2)$ NURBS surface elements are utilised. It is clearly observed that the difference between the NURBS IGA/Membrane and the NURBS IGA/Shell discretisations can be considered negligible, regardless of the mesh density, which means the membrane stiffness is much larger than its bending counterpart. Consequently, in the following two examples the membrane structures will only account for membrane stiffness, therefore neglecting the effects of the bending stiffness.

\subsubsection{Mesh convergence study}

A mesh convergence analysis is carried out in order to demonstrate the stability of the present membrane analysis. Figure $\mathbf{5}$ illustrates the convergence status of the maximum deflections of the membrane at steady state under $k$-refinement on meshes using the NURBS IGA/Membrane model. Additionally, the data corresponding to Figure 5 are tabulated in Table 1. As before, quadratic $(p=2)$ NURBS surface elements are still employed here. Clearly, although the coarsest mesh of $3 \times 3$ elements exhibits some locking, both the excellent accuracy and the general trend towards convergence are noteworthy in this figure. Furthermore, one can clearly observe that the maximum deflection properly converges to the corresponding reference solution as mesh refinement for the NURBS IGA/Shell method is increased, with a convergence rate $\left(R_{c}\right)$, in terms of the error of $L^{2}$-norm of maximum deflection $\left(e_{d}\right)$ against the NURBS element size $h_{e}$, being approximately 2.0. This demonstrates one salient property, as noted in section 4.2, of the $C^{k}(k \geq 1)$ continuity for the NURBS IGA.

\subsubsection{Accuracy study}

In order to investigate the accuracy of the NURBS-based shell/membrane formulation, the maximum deflections of the membrane calculated from the numerical and theoretical models and obtained from the experiments are compared in Figure 6 and Table 4. A set of typical deformed contour plots of the membrane calculated using the NURBS IGA/Membrane model under a pressure $p_{\text {int }}=100 \mathrm{~Pa}$ is plotted on Figure 7. We compare the deflections at points $\mathrm{D}_{1}$ to $\mathrm{D}_{8}$ along the diagonal and $\mathrm{E}_{1}$ to $\mathrm{E}_{8}$ along the edge under a typical pressure $p_{\text {int }}=100 \mathrm{~Pa}$. The corresponding results are listed in Table 2-3 and shown in Figure 8. The characteristics of the different computational, analytical and experimental models are summarised below:

- NURBS IGA/Membrane and IGA/Shell with $6 \times 6$ quadratic elements of (92 DOFs);

- In-house FEM/Membrane with triangular elements (7842 DOFs);

- ABAQUS-based FEM/Shell with triangular S3 elements (C $\left.{ }^{0}\right)$ (7842 DOFs) [53];

- Theoretical model with (geometrically/materially) linear assumption

- Experimental observations [50].

The deflections from the NURBS IGA models are comparable to those of their FEM counterparts whatever the pressure applied, although the number of DOFs in the NURBS IGA case is only $1 / 80$ of the that in the FEM models. It is noteworthy that for the 50, 100 and $150 \mathrm{~Pa}$ pressures the present NURBS IGA models apparently perform better than the FEM models.

The deflections from the numerical models, considering geometric non-linearities, all closely approximate the corresponding experimental solutions when the pressure is large enough $p_{\text {int }}=50,100,150 \mathrm{~Pa}$, while the results from the linear theoretical model match the experimental ones well if $p_{\text {int }}=25 \mathrm{~Pa}$. These findings suggest that it is necessary to account for the large deformation to simulate membrane structures, which is also in accordance to the objective of the present work. 
It should be emphasised that the computational cost per degree of freedom was not compared since the implementations are drastically different. It is however expected that the NURBS-based elements are more expensive than their Lagrange counterparts.

\subsubsection{Effect of surface order}

The influence of NURBS surface representation order is also investigated. Figure 9 presents convergence of the maximum deflection against the surface order $p=2,3$ and 4 for $3 \times 3$ NURBS elements under a pressure of $p_{\text {int }}=100 \mathrm{~Pa}$, and the data are listed in Table 5. Evidently, the trend towards convergence with increase of surface order is clearly exhibited.

\subsection{Example 2 - Inflation of a spherical membrane structure under internal pressure}

The next example consists of the dynamic inflation of a spherical membrane (radius $r=1.0 \mathrm{~m}$ ) under a uniform internal pressure (Figure 10-a). The material properties are as follows: Young's modulus $E=10^{4} \mathrm{~Pa}$, Poisson's ratio $v=0.2$, density $\rho=1 \times 10^{3} \mathrm{~kg} / \mathrm{m}^{3}$ and damping coefficient $\mu=5 \mathrm{~N} \cdot \mathrm{s} /(\mathrm{kg} \cdot \mathrm{m})$. The membrane is assumed to be at rest in the undeformed configuration (null initial velocity everywhere). The membrane thickness is assumed to have a constant value $h=0.001 \mathrm{~m}$. A uniform interior pressure $p_{\text {int }}(t)$ is applied on the inner surface of the structure in the following fashion:

$$
p_{\text {int }}(t)=\left\{\begin{array}{cl}
k t & k t<p_{\max } \\
p_{\max } & k t \geq p_{\max }
\end{array}\right.
$$

where $k=1 \mathrm{~Pa} / \mathrm{s}$ and $p_{\max }=3 \mathrm{~Pa}$. Owing to symmetry, only one octant of the specimen is used in the computations. The geometry, loading and boundary conditions are shown in Figure 10-b. A constant time step $\Delta t=10^{-3} \mathrm{~s}$ is employed. Meshes of $3 \times 3,6 \times 6$, and $12 \times 12$ quadratic NURBS surface elements are employed to analyse the finite deformation process of membrane inflation. For comparison, this case is also replicated using the standard FEM with 192, 3,072 and 49,152 3-noded membrane elements.

Figure 11 displays the initial and deformed configurations of the spherical membrane structure at time $t=2 \mathrm{~s}$ and $t=25 \mathrm{~s}$ (steady-state) solved by the above numerical models with different mesh settings. Table 6 lists the computed average radii $r$ at steady state for different mesh configurations. The pressure driven NURBS IGA solutions are clearly comparable to those obtained using a standard Lagrange-based interpolation for the discretisation, although the average radii for the NURBS IGA seems to be converging to a value that is over $5 \%$ greater than that computed by Lagrange-based FEM, probably due to the "over-stiff" behaviour of linear 3-node triangular elements employed in the Lagrange-based FEM.

It is worth noting that, much fewer DOFs are employed in the NURBS IGA computations to reach a similar degree of mesh convergence (Figure 11 and Figure 12). The clear advantage of NURBS IGA ability to capture exact geometries independently of the level of mesh refinement is in stark contrast with the traditional FEM approaches (Figure 11).

\subsection{Example 3 - Inflation of a square airbag}

In this example, we apply the NURBS IGA membrane element to the problem of inflation of a square airbag (with an edge length $a=1 \mathrm{~m}$ and a $10^{-5} \mathrm{~m}$ membrane thickness) (Figure 13). The material properties are: Young's modulus $E=10^{4} \mathrm{~Pa}$, Poisson ratio $v=0.0$, density $\rho=1 \times 10^{4} \mathrm{~kg} / \mathrm{m}^{3}$ and damping coefficient $\mu=0.5 \mathrm{~N} \cdot \mathrm{s} /(\mathrm{kg} \cdot \mathrm{m})$. The membrane is assumed to be at rest in the undeformed configuration (null initial velocity everywhere). The functional form of the internal pressure $p_{\text {int }}(t)$ applied to the internal surface of the airbag is the same as that described in the previous section (equation (77)) where $k=0.01 \mathrm{~Pa} / \mathrm{s}$ and $p_{\max }=0.01 \mathrm{~Pa}$. The steady-state solutions are achieved using the previously described dynamic relaxation scheme with a constant time step $\Delta t=10^{-3} \mathrm{~s}$. As for the previous example, meshes of $3 \times 3,6 \times 6$, and $12 \times 12$ quadratic NURBS surface elements are employed to analyse the problem of inflation of the square airbag. Again, the solutions calculated using FEM 3-noded membrane element models featuring 2,784, 6,192, and 17,304 elements are provided for comparison with the NURBS IGA Membrane models.Figure 14 shows the deformed shape of the square airbag at different stages of the inflation process, solved by the NURBS IGA with $6 \times 6$ quadratic surface elements. 
The deformed configurations clearly show the local buckling of the membrane surface due to the in-plane compression, which leads to the wrinkles that appear at the edges of the airbag. Figure $\mathbf{1 5}$ plots the deformed shapes of the square airbag at steady state for different mesh configurations $(3 \times 3,6 \times 6$, and $12 \times 12$ ). As intuitively expected, coarse meshes inhibit wrinkle formation. Conversely, mesh refinement is accompanied by an increase in the fineness (increase frequency) of the wrinkle pattern. The characteristic wavelength of the wrinkles is related to the element size, i.e., smaller element size produces smaller wavelength of the wrinkles. This phenomenon was also observed in various studies $[16,17,20,45,54]$.

By way of example, Figure 16 shows the computed maximum displacement at the centre of the airbag as a function of the number of degrees of freedom. It is found again that the maximum deflection quickly reaches convergence as the number of degrees of freedom is increased. Table 7 compares the computed maximum displacement at steady state for the NURBS IGA and the FEM models, with different mesh configurations. The good agreement between the two solutions is evident from this table.

\subsection{Example 4 - Inflation of a hyperelastic balloon}

We conclude this section with an example of the inflation of a rubber hyperelastic ballon, loaded by an internal pressure $p_{\text {int }}$. This problem, which shows an instability, is amenable to analytical solution [55], and, therefore, provides a convenient basis for the assessment of the present NURBS IGA membrane element in handling hyperelasticity. Due to the equibiaxial deformation, two of the principal stretches lay on the plane tangential to the sphere and are equal to the radial stretch: $\lambda=\lambda_{1}=\lambda_{2}={ }^{0} R / R$, where ${ }^{0} R$ is the radius of the undeformed middle surface of the shell, $R$ is the corresponding radius for the deformed shell. The relation between the internal pressure and the radial stretch are then given by

$$
p_{\text {int }}=2 \frac{{ }^{0} H}{{ }^{0} R} \sigma_{\theta}=2 \frac{{ }^{0} H}{{ }^{0} R \lambda^{2}} \frac{\partial W}{\partial \lambda}
$$

Where $H$ and ${ }^{0} H$ are the current and initial balloon thicknesses, respectively, $\sigma_{\theta}$ corresponds to the hoop stress, and $W$ is the strain-energy density of the material per unit undeformed volume. Substituting equation (55) into (78) yields the closed-form solution:

$$
p_{\text {int }}=2 \frac{{ }^{0} H}{{ }^{0} R} \sum_{r} \mu_{r}\left(\lambda^{\alpha_{r}-3}-(\lambda)^{-2 \alpha_{r}-3}\right)
$$

We consider a balloon with initial radius ${ }^{0} R=100 \mathrm{~mm}$ and initial thickness ${ }^{0} H=1 \mathrm{~mm}$ composed of a three-term Ogden model with material constants provided below:

$$
\begin{array}{ll}
\mu_{1}=630 \mathrm{kPa} & \alpha_{1}=1.3 \\
\mu_{2}=1.2 \mathrm{kPa} & \alpha_{2}=5.0 \\
\mu_{3}=-10 \mathrm{kPa} & \alpha_{3}=-2.0
\end{array}
$$

Other material parameters are density $\rho=1 \times 10^{3} \mathrm{~kg} / \mathrm{m}^{3}$ and damping coefficient $\mu=5 \mathrm{~N} \cdot \mathrm{s} /(\mathrm{kg} \cdot \mathrm{m})$ Owing to symmetry, only one octant of the specimen is used in the computations. When an internal pressure of $p_{\text {int }}=5 \mathrm{kPa}$ is applied, the analytical value of the radial stretch is 1.24 from equation (79). A constant time step $\Delta t=10^{-3} \mathrm{~s}$ is employed. As before, meshes of $3 \times 3,6 \times 6$, and $12 \times 12$ quadratic NURBS surface elements are employed to analyse the finite deformation process of this hyperelastic balloon inflation. Figure 17 shows the computed radial stretch against the number of degrees of freedom. The good accuracy and the general trend towards convergence are clearly found in this figure.

\section{Conclusions}

An explicit NURBS-based isogeometric (NURBS IGA) framework for thin structures complying with the Kirchhoff-Love assumption was developed to solve the static/dynamic equilibrium equations for membrane structures undergoing arbitrary deformations. Both membrane and bending stiffnesses are accounted for in the theoretical and computational formulations so that the framework is applicable to thin to ultra-thin shells and pure membranes. The displacement field is interpolated from the control points of the NURBSbased mid-surface of the thin structure. The normal to the mid-surface is fully determined from the surface curvilinear coordinates of the mid-surface so that no rotational degrees of freedom are required. 
The dynamic relaxation method was shown to be an efficient means to obtain steady state solutions whilst benefiting of the advantages of an explicit scheme in dealing with highly geometrically non-linear problems. Through the formulations and numerical examples, the following conclusions can be drawn:

(1) The NURBS IGA solutions are found to be comparable to those of their FEM counterparts, particularly for large deformation of membrane structures, with the advantage of a much reduced number of degrees of freedom and an exact geometry independent of the NURBS mesh.

(2) Due to the high order $C^{k}$ continuity $(k \geq 1)$ in NURBS shape functions, the non-linear IGA solutions are guaranteed to converge with mesh $k$-refinement, thereby side-stepping the difficulties associated with the use of $C^{0}$ methods.

(3) Analysis of NURBS-based thin shell/membrane structures can be readily done for complex geometries (provided they lend themselves to a tensor product form within a multi-patch framework) due to the direct link between geometry and discretisation that does not require any time-consuming (i.e. human-operated) meshing process compared to the traditional FEM approaches.

(4) The solutions to the airbag inflation problem presented here highlighted the potential offered by isogeometric membrane formulations to simulate wrinkling and folding of membrane structures which have been widely observed in Nature and have recently received much attention in the literature [5, 56-60]. Addressing the dynamics of biological membranes is also key in unravelling biomimetic principles. For example, the hydrodynamic motion strategies of microorganisms such as euglenoids which rely on oscillatory deformations of their enclosing membrane could lead to the development of engineered "micro-swimmers" [61].

(5) A simple non-linear elastic material model (Ogden's formulation) was implemented in the present IGA framework. The implementation of more suitable anisotropic hyperelastic models to represent the passive and adaptive behaviour of biological membrane structures $[10,55,62,63]$ in the present IGA framework is under way.

\section{Acknowledgements}

Georges Limbert and Lei Chen would like to acknowledge the financial support from the European Office of Aerospace Research and Development (Air Force Office of Scientific Research) [Grant FA8655-12-1-2103] and the Engineering and Physical Sciences Research Council (EPSRC) [Grant EP/F034296/1]. Stéphane Bordas and Timon Rabczuk would like to acknowledge the partial financial support of the Framework Programme 7 Initial Training Network Funding under grant number 289361 "Integrating Numerical Simulation and Geometric Design Technology".

Stéphane Bordas would like to thank partial funding for his time from: 1) the European Research Council Starting Independent Research Grant (ERC Stg grant agreement No. 279578) entitled "Towards real time multiscale simulation of cutting in non-linear materials with applications to surgical simulation and computer guided surgery; 2) the EPSRC under grant EP/G042705/1 Increased Reliability for Industrially Relevant Automatic Crack Growth Simulation with the eXtended Finite Element Method. 


\section{References}

[1] Wempner, G. \& TAlaslidis, D. 2002. Mechanics of Solids and Shells: Theories and approximations, CRC Press.

[2] Jenkins, C. H. \& LeONARD, J. W. 1991. Nonlinear dynamic response of membranes: state of the art. Applied Mechanics Review, 44 (7), 319-328.

[3] LeE, E. S. \& Youn, S. K. 2006. Finite element analysis of wrinkling membrane large deformations. Finite Elements in Analysis and Design, 42 (8-9), 780-791.

[4] Rossi, R., Lazzari, M., Vitaliani, R. \& Onate, E. 2005. Simulation of light-weight membrane structures by wrinkling model. International Journal for Numerical Methods in Engineering, 62 (15), 2127-2153.

[5] Cerda, E. \& Mahadevan, L. 2003. Geometry and physics of wrinkling. Physical Review Letters, 90 (7), 074302-1:074302-4.

[6] Belytschko, T., Stolarski, H., Liu, W. K., Carpenter, N. \& Ong, J. S. J. 1985. Stress projection for membrane and shear locking in shell finite elements. Computer Methods in Applied Mechanics and Engineering, 51 (1-3), 221-258.

[7] Simo, J. C., Fox, D. D. \& RIfAI, M. S. 1989. On a Stress Resultant Geometrically Exact ShellModel .2. The Linear-Theory - Computational Aspects. Computer Methods in Applied Mechanics and Engineering, 73 (1), 53-92.

[8] Simo, J. C. \& Fox, D. D. 1989. On a Stress Resultant Geometrically Exact Shell-Model .1. Formulation and Optimal Parametrization. Computer Methods in Applied Mechanics and Engineering, 72 (3), 267-304.

[9] Prot, V., Skallerud, B. \& Flolzapfel, G. A. 2007. Transversely isotropic membrane shells with application to mitral valve mechanics. Constitutive modelling and finite element implementation. International Journal for Numerical Methods in Engineering, 71 (8), 987-1008.

[10] Rausch, M. K., Tibayan, F. A., Miller, D. C. \& Kuhl, E. 2012. Evidence of adaptive mitral leaflet growth. Journal of the Mechanical Behavior of Biomedical Materials, 15, 208-217.

[11] Rahimi, M. \& ARroyo, M. 2012. Shape dynamics, lipid hydrodynamics, and the complex viscoelasticity of bilayer membranes. Physical Review E, 86 (1).

[12] Bathe, K. J. 1982. Finite element procedures in engineering analysis, Englewood Cliffs, PrenticeHall, Inc.

[13] Belytschko, T., Lin, J. I. \& Tsay, C. S. 1984. Explicit algorithms for the nonlinear dynamics of shells. Computer Methods in Applied Mechanics and Engineering, 42 (2), 225-251.

[14] Spillers, W. R., Schlogel, M. \& Pilla, D. 1992. A simple membrane finite element. Computers \& Structures, 45 (1), 181-183.

[15] Bonet, J., Wood, R. D., Mahaney, J. \& Heywood, P. 2000. Finite element analysis of air supported membrane structures. Computer Methods in Applied Mechanics and Engineering, 190 (57), 579-595.

[16] Tsiatas, G. C. \& Katsikadelis, J. T. 2006. Large deflection analysis of elastic space membranes. International Journal for Numerical Methods in Engineering, 65 (2), 264-294.

[17] Wu, T. Y. \& TiNG, E. C. 2008. Large deflection analysis of 3D membrane structures by a 4-node quadrilateral intrinsic element. Thin-Walled Structures, 46 (3), 261-275.

[18] Hughes, T. J. R., Cottrell, J. A. \& Bazilevs, Y. 2005. Isogeometric analysis: CAD, finite elements, NURBS, exact geometry and mesh refinement. Computer Methods in Applied Mechanics and Engineering, 194 (39-41), 4135-4195.

[19] Cottrell, J. A., Hughes, T. J. R. \& Bazilevs, Y. 2009. Isogeometric analysis: toward integration of $C A D$ and FEA, Oxford, Wiley.

[20] CiraK, F. \& OrTiZ, M. 2001. Fully $\mathrm{C}^{1}$-conforming subdivision elements for finite deformation thinshell analysis. International Journal for Numerical Methods in Engineering, 51 (7), 813-833.

[21] CiraK, F., ORtiz, M. \& SCHRÖDER, P. 2000. Subdivision surfaces: a new paradigm for thin-shell finite-element analysis. International Journal for Numerical Methods in Engineering, 47 (12), 20392072.

[22] RABCZUK, T. \& AREias, P. M. A. 2006. A meshfree thin shell for arbitrary evolving cracks based on an external enrichment. Computer Modeling in Engineering and Sciences, 16 (2), 115-130. 
[23] Rabczuk, T., Areias, P. M. A. \& Belytschko, T. 2007. A meshfree thin shell method for nonlinear dynamic fracture. International Journal for Numerical Methods in Engineering, 72 (5), 524548.

[24] Rabczuk, T., Gracie, R., Song, J.-H. \& Belytschko, T. 2010. Immersed particle method for fluid-structure interaction. International Journal for Numerical Methods in Engineering, 81 (1), 4871.

[25] Millán, D., Rosolen, A. \& Arroyo, M. 2011. Thin shell analysis from scattered points with maximum-entropy approximants. International Journal for Numerical Methods in Engineering, 85 (6), 723-751.

[26] Millán, D., Rosolen, A. \& Arroyo, M. 2013. Nonlinear manifold learning for meshfree finite deformation thin-shell analysis. International Journal for Numerical Methods in Engineering, 93 (7), 685-713.

[27] Piegl, L. \& TilLer, W. 1997. The NURBS Book, New York, Springer-Verlag.

[28] BAZILEVS, Y. \& Hughes, T. J. R. 2008. NURBS-based isogeometric analysis for the computation of flows about rotating components. Computational Mechanics, 43 (1), 143-150.

[29] Cottrell, J. A., Reali, A., Bazilevs, Y. \& Hughes, T. J. R. 2006. Isogeometric analysis of structural vibrations. Computer Methods in Applied Mechanics and Engineering, 195 (41-43), 52575296.

[30] Bazilevs, Y., Calo, V. M., Hughes, T. J. R. \& Zhang, Y. 2008. Isogeometric fluid-structure interaction: theory, algorithms, and computations. Computational Mechanics, 43 (1), 3-37.

[31] Bazilevs, Y., Calo, V. M., Zhang, Y. \& Hughes, T. J. R. 2006. Isogeometric fluid-structure interaction analysis with applications to arterial blood flow. Computational Mechanics, 38 (4-5), 310322 .

[32] Hughes, T. J. R., Reali, A. \& Sangaldi, G. 2008. Duality and unified analysis of discrete approximations in structural dynamics and wave propagation: Comparison of p-method finite elements with k-method NURBS. Computer Methods in Applied Mechanics and Engineering, 197 (49-50), 4104-4124.

[33] Benson, D. J., Bazilevs, Y., Hsu, M. C. \& Hughes, T. J. R. 2010. Isogeometric shell analysis: The Reissner-Mindlin shell. Computer Methods in Applied Mechanics and Engineering, 199 (5-8), 276289.

[34] Benson, D. J., Bazilevs, Y., Hsu, M. C. \& Hughes, T. J. R. 2011. A large deformation, rotationfree, isogeometric shell. Computer Methods in Applied Mechanics and Engineering, 200 (13-16), 1367-1378.

[35] Wall, W. A., Frenzel, M. A. \& CYron, C. 2008. Isogeometric structural shape optimization. Computer Methods in Applied Mechanics and Engineering, 197 (33-40), 2976-2988.

[36] Hassani, B., Tavakkoli, S. M. \& Moghadam, N. Z. 2011. Application of isogeometric analysis in structural shape optimization. Scientia Iranica, 18 (4), 846-852.

[37] Bazilevs, Y., Calo, V. M., Cottrell, J. A., Evans, J. A., Hughes, T. J. R., Lipton, S., Scott, M. A. \& Sederberg, T. W. 2010. Isogeometric analysis using T-splines. Computer Methods in Applied Mechanics and Engineering, 199 (5-8), 229-263.

[38] Dorfel, M. R., Juttler, B. \& Simeon, B. 2010. Adaptive isogeometric analysis by local hrefinement with T-splines. Computer Methods in Applied Mechanics and Engineering, 199 (5-8), 264-275.

[39] Nguyen-Thanh, N., Nguyen-Xuan, H., Bordas, S. P. A. \& Rabczuk, T. 2010. Isogeometric finite element analysis using polynomial splines over hierarchical T-meshes. IOP Conference Series Materials Science and Engineering, 10(1) (1), Doi 10.1088/1757-899x/10/1/012238.

[40] Nguyen-Thanh, N., Nguyen-Xuan, H., Bordas, S. P. A. \& Rabczuk, T. 2011. Isogeometric analysis using polynomial splines over hierarchical T-meshes for two-dimensional elastic solids. Computer Methods in Applied Mechanics and Engineering, 200 (21-22), 1892-1908.

[41] Nguyen-Thanh, N., Kiendl, J., Nguyen-Xuan, H., Wuchner, R., Bletzinger, K. U., Bazilevs, Y. \& RABCZUK, T. 2011. Rotation free isogeometric thin shell analysis using PHT-splines. Computer Methods in Applied Mechanics and Engineering, 200 (47-48), 3410-3424.

[42] Kiende, J., Bletzinger, K. U., Linhard, J. \& Wuchner, R. 2009. Isogeometric shell analysis with Kirchhoff-Love elements. Computer Methods in Applied Mechanics and Engineering, 198 (49-52), 3902-3914.

[43] Kiende, J., Bazilevs, Y., Hsu, M. C., Wuchner, R. \& Bletzinger, K. U. 2010. The bending strip method for isogeometric analysis of Kirchhoff-Love shell structures comprised of multiple patches. Computer Methods in Applied Mechanics and Engineering, 199 (37-40), 2403-2416. 
[44] Belytschko, T., Liu, W. K. \& Moran, B. 2000. Nonlinear Finite Elements for Continua and Structures, Oxford, Wiley.

[45] Haseganu, E. M. \& Steigmann, D. J. 1994. Analysis of partly wrinkled membranes by the method of dynamic relaxation. Computational Mechanics, 14 (6), 596-614.

[46] Sauve, R. G. \& MetzGer, D. R. 1995. Advances in dynamic relaxation techniques for nonlinear finite element analysis. Journal of Pressure Vessel Technology-Transactions of the ASME, 117 (2), 170-176.

[47] Nguyen, N. P., Simpson, R. N., Bordas, S. P. A. \& Rabczuk, T. 2013. An introduction to Isogeometric Analysis with Matlab implementation: FEM and XFEM formulations. Available: http://arxiv.org/abs/1205.2129.

[48] OGDen, R. W. 1984. Non-Linear Elastic Deformations, West Sussex, England, Ellis Horwood Ltd.

[49] Marsden, J. E. \& Hughes, T. J. R. 1994. Mathematical Foundations of Elasticity, New-York, Dover.

[50] ShI, X. \& BurnetT, E. 2008. Mechanics and test study of flexible membranes ballooning in three dimensions. Building and Environment, 43 (11), 1871-1881.

[51] Shi, X., LiAng, J. \& BurnetT, E. 2006. Mechanics and test study of two-dimensional flexible membranes. Journal of Architectural Engineering, 12 (2), 93-99.

[52] Wang, S., LiU, G. R., Zhang, Z. Q. \& Chen, L. 2011. Nonlinear 3D numerical computations for the square membrane versus experimental data. Engineering Structures, 33 (5), 1828-1837.

[53] 2012. ABAQUS Theory Manual, Providence, RI, USA, Simulia, Dassault Systèmes.

[54] Zhang, Z. Q. \& LiU, G. R. 2011. An edge-based smoothed finite element method (ES-FEM) using 3node triangular elements for 3D non-linear analysis of spatial membrane structures. International Journal for Numerical Methods in Engineering, 86 (2), 135-154.

[55] Holzapfel, G. A. 2000. Nonlinear Solid Mechanics. A Continuum Approach for Engineering, Chichester, UK, John Wiley \& Sons.

[56] LI, B., CAO, Y. P., FEnG, X. Q. \& GAO, H. J. 2012. Mechanics of morphological instabilities and surface wrinkling in soft materials: a review. Soft Matter, 8 (21), 5728-5745.

[57] Genzer, J. \& Groenewold, J. 2006. Soft matter with hard skin: From skin wrinkles to templating and material characterization. Soft Matter, 2 (4), 310-323.

[58] LiDmar, J., MiRny, L. \& Nelson, D. R. 2003. Virus shapes and buckling transitions in spherical shells. Physical Review E, 68 (5), -.

[59] Bernal, R., Tassius, C., Melo, F. \& Geminard, J. C. 2007. Mechanical characterization of elastic membranes: Cell mechanics applications. Applied Physics Letters, 90 (6), --

[60] Dervaux, J., Ciarletta, P. \& Ben Amar, M. 2009. Morphogenesis of thin hyperelastic plates: A constitutive theory of biological growth in the Foppl-von Karman limit. Journal of the Mechanics and Physics of Solids, 57 (3), 458-471.

[61] Arroyo, M., Heltai, L., Millán, D. \& DeSimone, A. 2012. Reverse engineering the euglenoid movement. Proceedings of the National Academy of Sciences, 109 (44), 17874-17879.

[62] ZÖLlner, A. M., Buganza Tepole, A. \& KuHL, E. 2012. On the biomechanics and mechanobiology of growing skin. Journal of Theoretical Biology, 297, 166-175.

[63] LIMBERT, G. 2011. A mesostructurally-based anisotropic continuum model for biological soft tissues-Decoupled invariant formulation. Journal of the Mechanical Behavior of Biomedical Materials, 4 (8), 1637-1657. 
Table 1. Mesh sensitivity: maximum deflections $(H: \mathrm{mm})$ of the square membrane converge with $k$ refinement on meshes.

\begin{tabular}{|c|c|c|c|c|c|}
\hline \multicolumn{2}{|c|}{ Mesh: number of NURBS elements } & $3 \times 3$ & $6 \times 6$ & $12 \times 12$ & $24 \times 24$ \\
\hline \multirow{3}{*}{$\begin{array}{l}\text { Maximum deflection } \\
H: \mathrm{mm}, p=25 \mathrm{~Pa}\end{array}$} & NURBS IGA/Membrane & 11.9 & 12.9 & 13.1 & 13.2 \\
\hline & NURBS IGA/Shell & 11.9 & 12.9 & 13.0 & 13.1 \\
\hline & Reference & \multicolumn{4}{|c|}{13.4} \\
\hline \multirow{3}{*}{$\begin{array}{l}\text { Maximum deflection } \\
H: \mathrm{mm}, p=50 \mathrm{~Pa}\end{array}$} & NURBS IGA/Membrane & 15.3 & 16.6 & 16.9 & 17.0 \\
\hline & NURBS IGA/Shell & 15.3 & 16.6 & 16.8 & 16.9 \\
\hline & Reference & \multicolumn{4}{|c|}{16.9} \\
\hline \multirow{3}{*}{$\begin{array}{l}\text { Maximum deflection } \\
H: \mathrm{mm}, p=100 \mathrm{~Pa}\end{array}$} & NURBS IGA/Membrane & 19.6 & 21.1 & 21.4 & 21.4 \\
\hline & NURBS IGA/Shell & 19.5 & 21.0 & 21.3 & 21.4 \\
\hline & Reference & \multicolumn{4}{|c|}{21.5} \\
\hline \multirow{3}{*}{$\begin{array}{l}\text { Maximum deflection } \\
H: \mathrm{mm}, \quad p=150 \mathrm{~Pa}\end{array}$} & NURBS IGA/Membrane & 22.6 & 24.4 & 24.9 & 25.1 \\
\hline & NURBS IGA/Shell & 22.5 & 24.2 & 24.7 & 25.0 \\
\hline & Reference & \multicolumn{4}{|c|}{25.4} \\
\hline
\end{tabular}

Table 2. Calculated diagonal deflections $(\mathrm{mm})$ of the square membrane under a pressure $p_{\text {int }}=100 \mathrm{~Pa}$.

\begin{tabular}{lllllllll}
\hline \hline Point: D & 1 & 2 & 3 & 4 & 5 & 6 & 7 & 8 \\
\hline NURBS IGA/Membrane & 3.9 & 7.5 & 11.2 & 14.2 & 17.1 & 19.6 & 20.8 & 21.1 \\
NURBS IGA/Shell & 3.9 & 7.5 & 11.2 & 14.2 & 17.1 & 19.5 & 20.7 & 21.0 \\
FEM/Membrane & 4.4 & 8.7 & 12.6 & 15.8 & 18.1 & 19.7 & 20.6 & 20.9 \\
FEM/Shell & 4.2 & 8.0 & 11.6 & 14.7 & 17.1 & 18.7 & 19.6 & 19.9 \\
Experiment [50] & 1.6 & 5.3 & 9.6 & 13.7 & 16.2 & 20.1 & 20.4 & 21.5 \\
\hline \hline
\end{tabular}

Table 3. Calculated edge deflections $(\mathrm{mm})$ of the square membrane under a pressure $p_{\text {int }}=100 \mathrm{~Pa}$.

\begin{tabular}{lllllllll}
\hline \hline Point: E & 1 & 2 & 3 & 4 & 5 & 6 & 7 & 8 \\
\hline NURBS IGA/Membrane & 2.6 & 5.6 & 8.9 & 11.8 & 14.1 & 15.7 & 16.7 & 17.0 \\
NURBS IGA/Shell & 2.6 & 5.6 & 8.9 & 11.8 & 14.1 & 15.6 & 16.6 & 16.9 \\
FEM/Membrane & 2.8 & 5.6 & 8.3 & 10.7 & 12.7 & 14.3 & 15.3 & 15.6 \\
FEM/Shell & 2.7 & 5.3 & 7.78 & 10.1 & 12.0 & 13.6 & 14.6 & 15.0 \\
Experiment [50] & 0.9 & 3.6 & 4.1 & 6.5 & 9.3 & 10.4 & 11.1 & 11.7 \\
\hline \hline
\end{tabular}


Table 4. Accuracy study for the four levels of internal pressure ( $p_{\text {int }}=25,50,100$ and $\left.150 \mathrm{~Pa}\right)$ : comparison of maximum deflections $(H: \mathrm{mm})$ of the square membrane obtained from the NURBS IGA models, Lagrange-based finite element models, Shi et al.'s [51] theoretical model and the physical experiments.

\begin{tabular}{llcccc}
\hline \hline Pressure: Pa & & 25 & 50 & 100 & 150 \\
\hline & NURBS IGA/Membrane (mesh: & 12.9 & 16.6 & 21.1 & 24.4 \\
& NURBS IGA/Shell (mesh: $9 \times 9)$ & 12.9 & 16.6 & 21.0 & 24.2 \\
Maximum & FEM/Membrane (mesh: 7842 & 13.2 & 16.6 & 20.9 & 23.9 \\
deflection $H:$ & FEM/Shell (mesh: 7842 DOFs) & 13.2 & 15.7 & 19.9 & 22.8 \\
mm & Shi et al. linear theory [51] & 8.8 & 11.2 & 14.1 & 16.1 \\
& Experiment [50] & $9.2 \pm 0.5$ & $17.6 \pm 0.9$ & $21.5 \pm 1.1$ & $23.8 \pm 1.2$ \\
\hline \hline
\end{tabular}

Table 5. Effect of surface order: maximum deflections $(H: \mathrm{mm})$ of the square membrane converge with the increase of surface order: $p=2,3$ and 4 for $3 \times 3$ NURBS elements.

\begin{tabular}{llccc}
\hline \hline Surface order & & $\mathrm{p}=2$ & $\mathrm{p}=3$ & $\mathrm{p}=4$ \\
Maximum & NURBS IGA/Membrane & 19.6 & 20.4 & 21.2 \\
deflection $H:$ & NURBS IGA/Shell & 19.5 & 20.3 & 21.0 \\
mm & Reference & & 21.5 & \\
\hline \hline
\end{tabular}

Table 6. Average radius of the deformed spherical membrane structure at steady state.

\begin{tabular}{cccccc}
\hline \hline \multicolumn{2}{c}{$\begin{array}{c}\text { NURBS IGA } \\
\text { (NURBS surface elements) }\end{array}$} & \multicolumn{3}{c}{ Lagrange-based FEM } \\
(T3-elements) \\
$3 \times 3$ & $6 \times 6$ & $12 \times 12$ & 192 & 3072 & 49125 \\
2.3506 & 2.3930 & 2.4108 & 2.1972 & 2.2578 & 2.2617 \\
\hline \hline
\end{tabular}

Table 7. Maximum vertical displacement of the deformed square airbag at steady state.

\begin{tabular}{|c|c|c|c|c|c|}
\hline \multicolumn{3}{|c|}{ NURBS IGA } & \multicolumn{3}{|c|}{ Lagrange-based FEM } \\
\hline \multicolumn{3}{|c|}{ (NURBS surface elements) } & \multicolumn{3}{|c|}{ (T3-elements) } \\
\hline $4 \times 4$ & $6 \times 6$ & $12 \times 12$ & 2714 & 6192 & 17304 \\
\hline 0.2213 & 0.2498 & 0.2555 & 0.2208 & 0.2401 & 0.2434 \\
\hline
\end{tabular}




\section{Physical space}

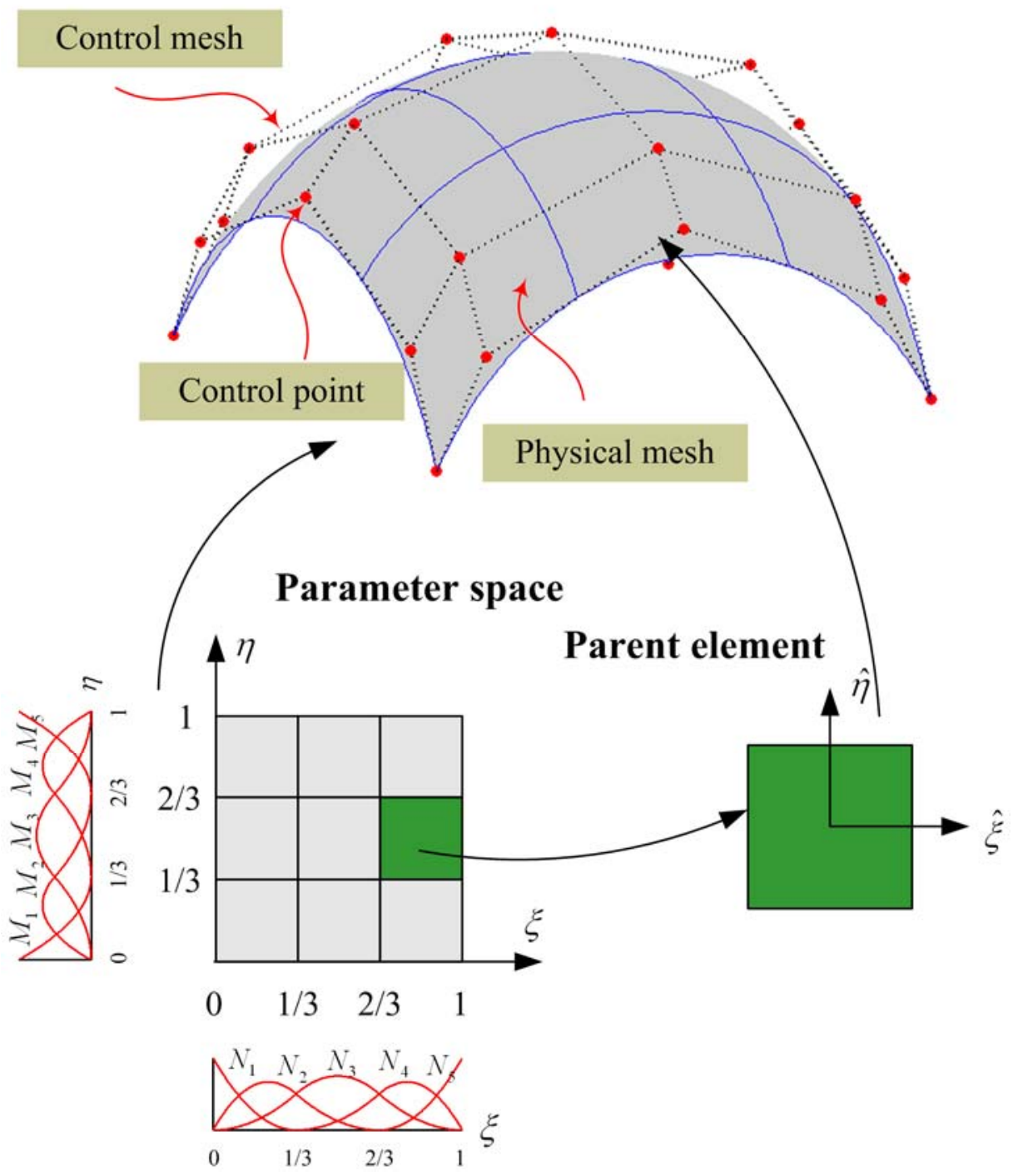

Figure 1. Physical mesh and control mesh for a cubic B-Spline surface. The control points (shown in red) defines the control polygon (dashed lines). Due to the open knot vectors the control points at the vertices of the surface are interpolated. The blue lines on the physical surface mark the knots which divide the surface into elements. 


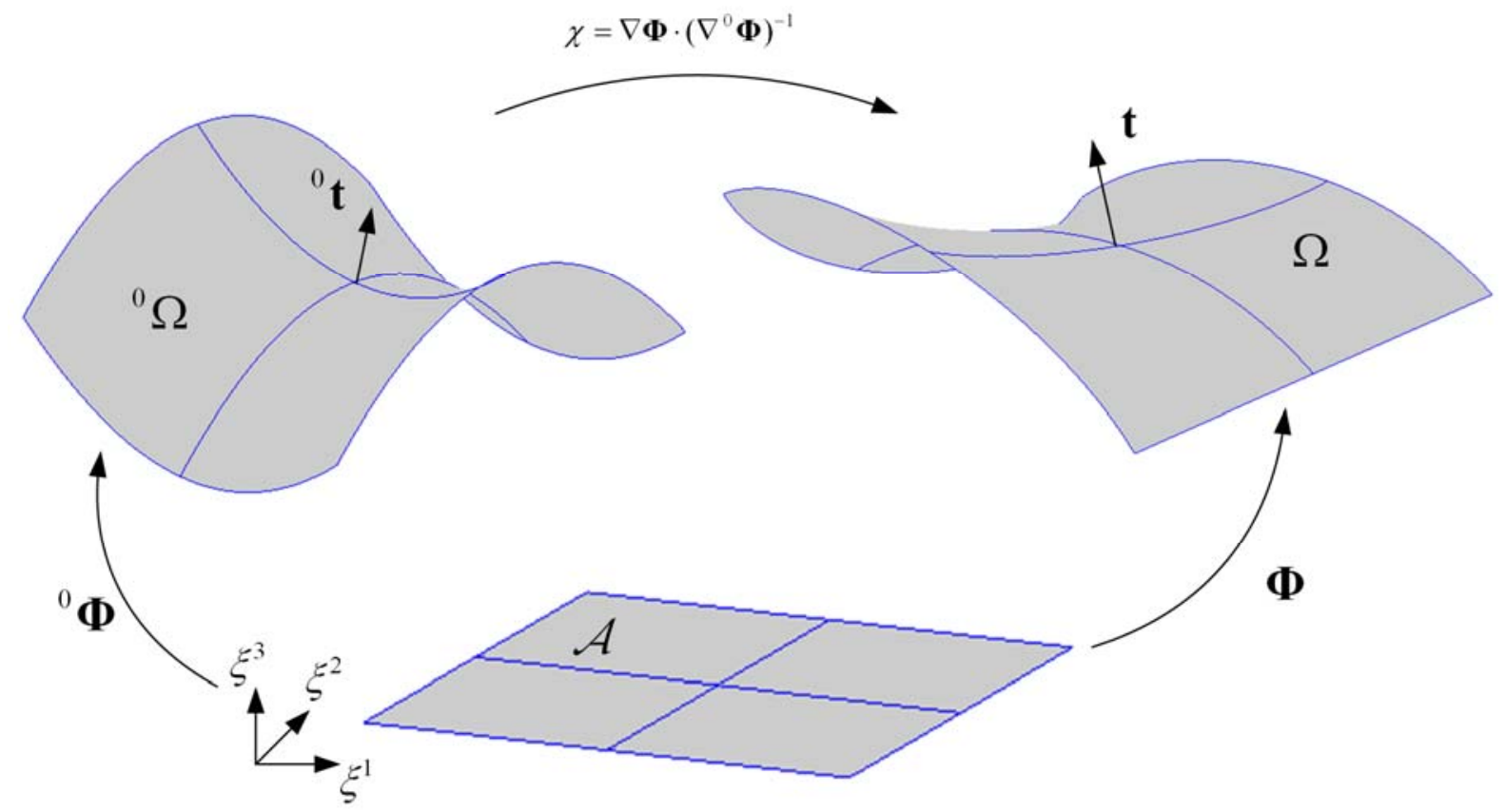

Figure 2. Kinematic description of a thin shell: schematic of geometry in the reference and the deformed configurations. 


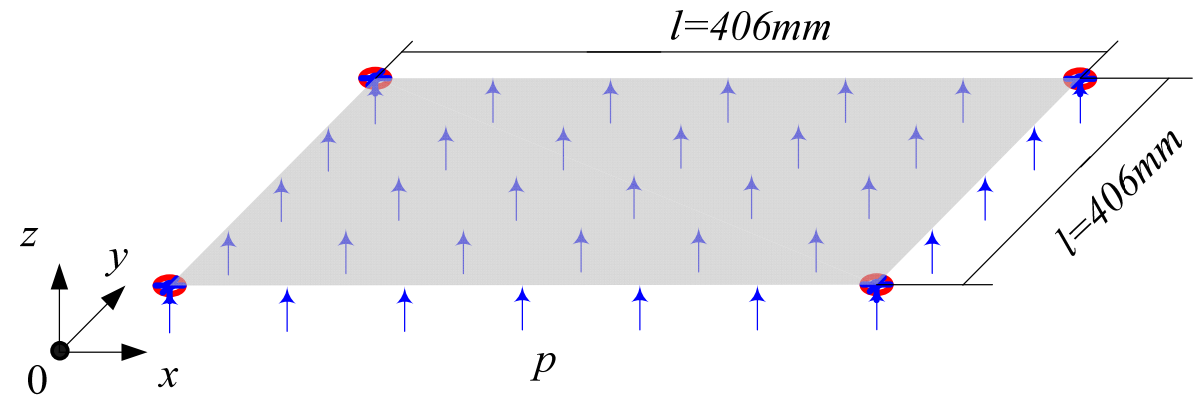

(a)

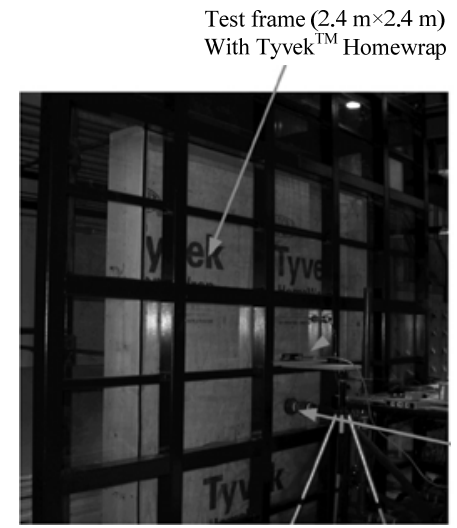

(b)

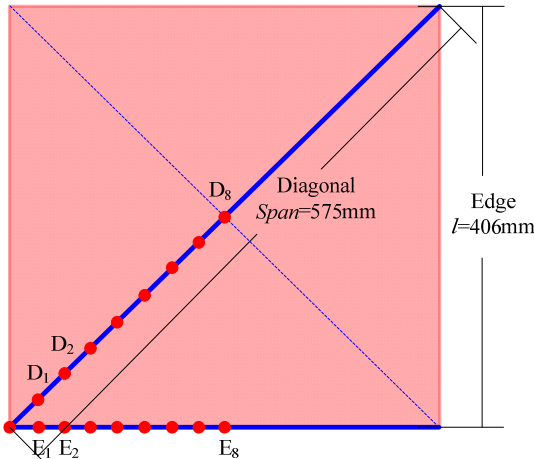

(c)

Figure 3. Problem setting of a square membrane subjected to a static pressure (a) geometry, loading and boundary conditions; (b) experimental setup in [50]; (c) displacement measurement configurations.

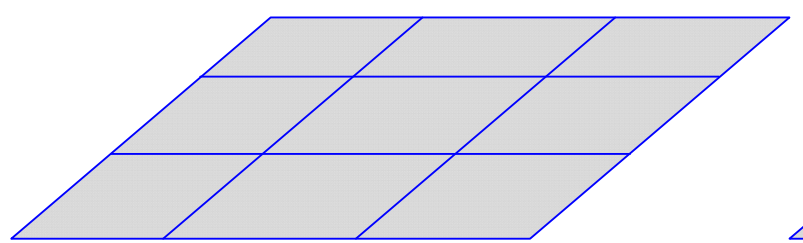

Mesh 1: $3 \times 3$

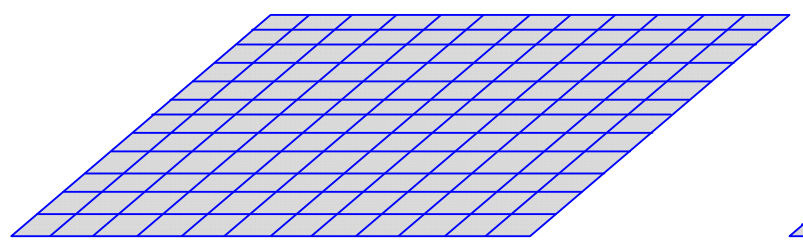

Mesh 3: $12 \times 12$

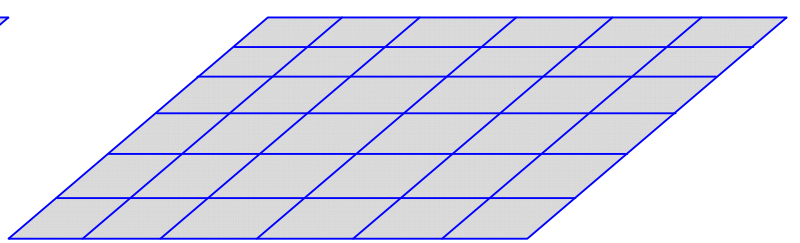

Mesh 2: 6×6

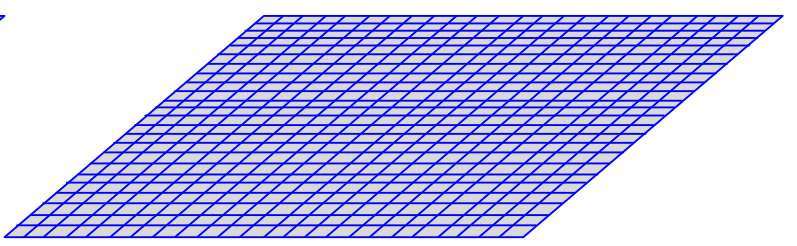

Mesh 4: $24 \times 24$

Figure 4. Undeformed meshes of the square membrane subjected to static pressure for the four level of $k$ refinement considered in the isogeometric analyses. 


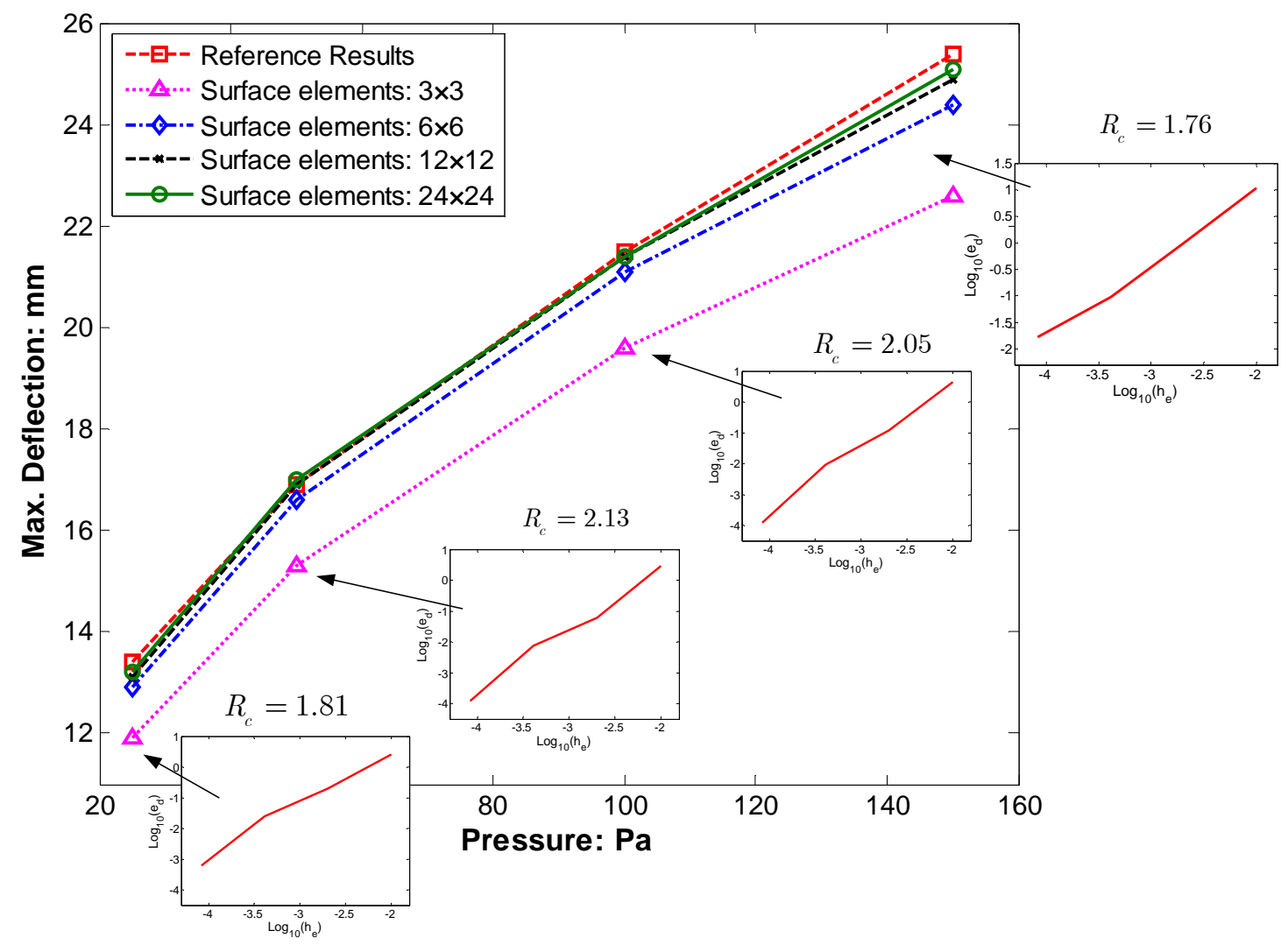

Figure 5. Results of the mesh sensitivity study (maximum deflection) for the square membrane under static pressure load.

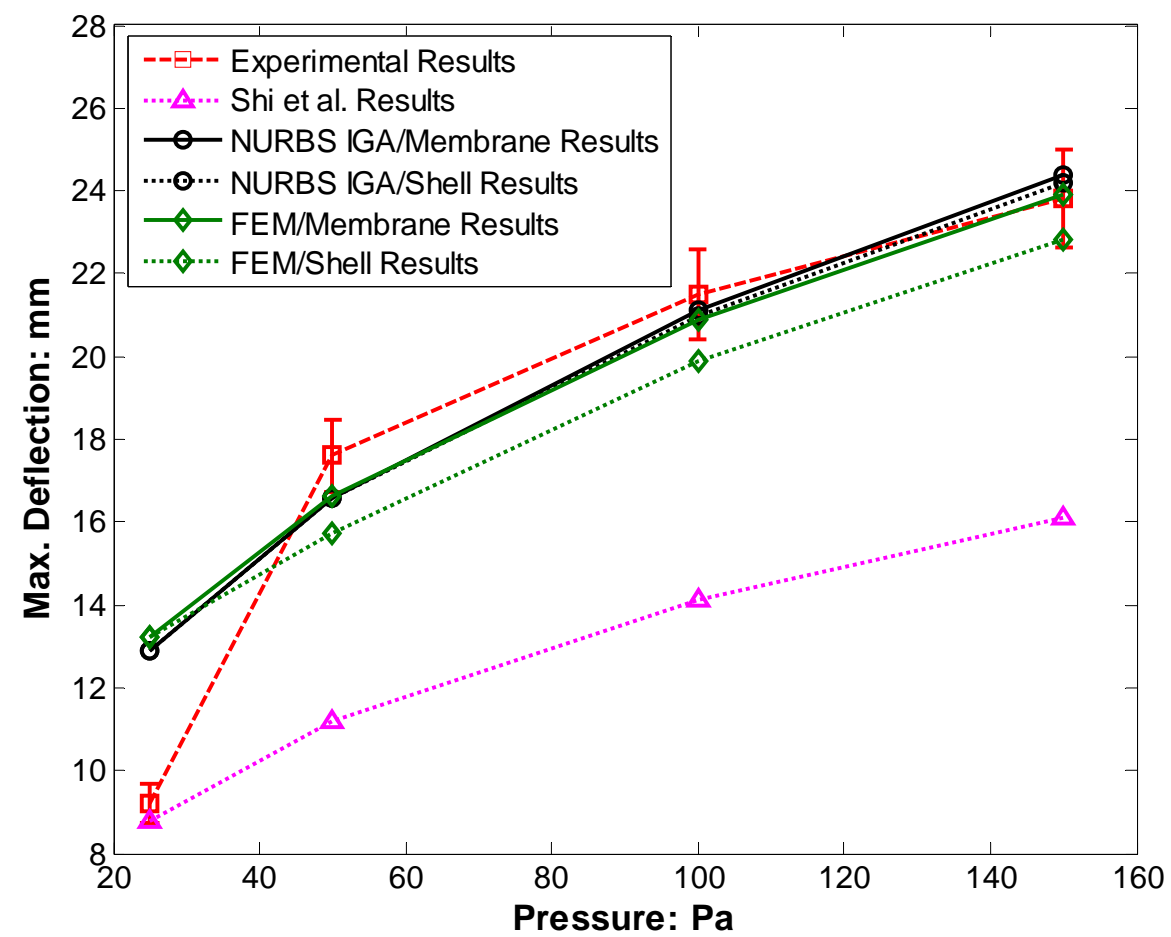

Figure 6. Results of the accuracy study (maximum deflection) for the square membrane under static pressure load.. 


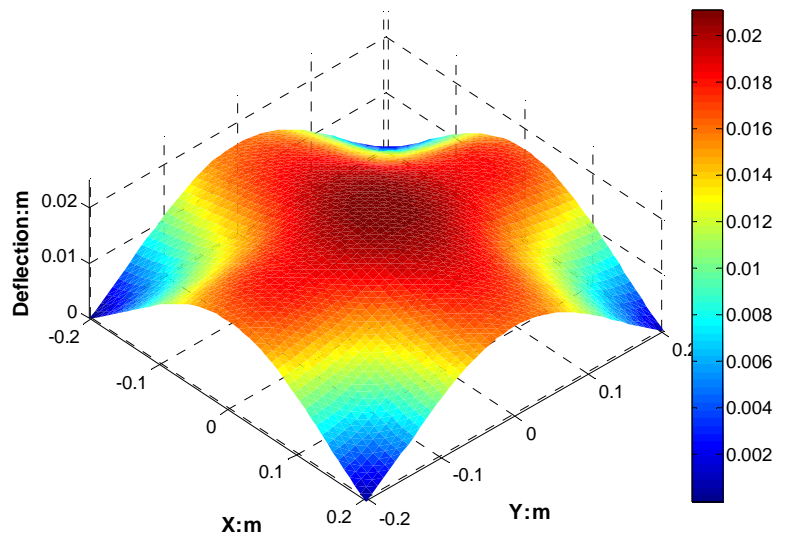

(a) Perspective view

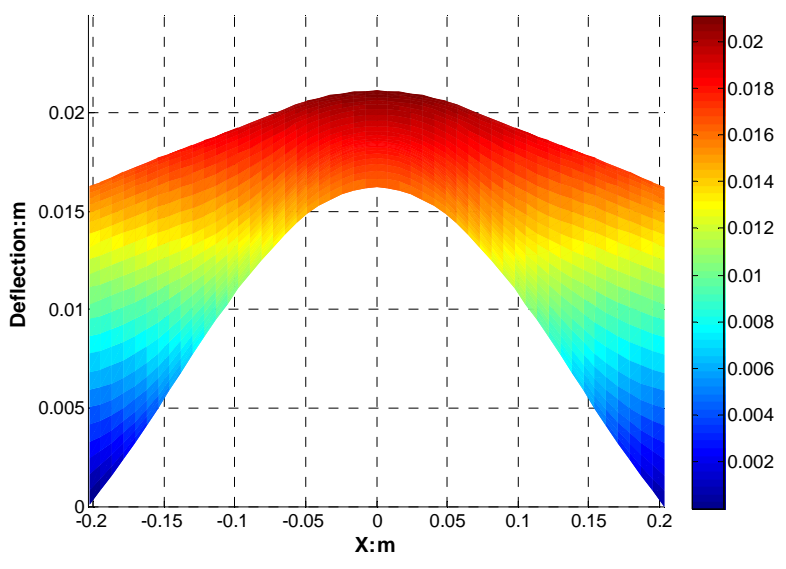

(b) Front view

Figure 7. Deformed profile of the square membrane under a static pressure of $p_{i n t}=100 \mathrm{~Pa}$. 


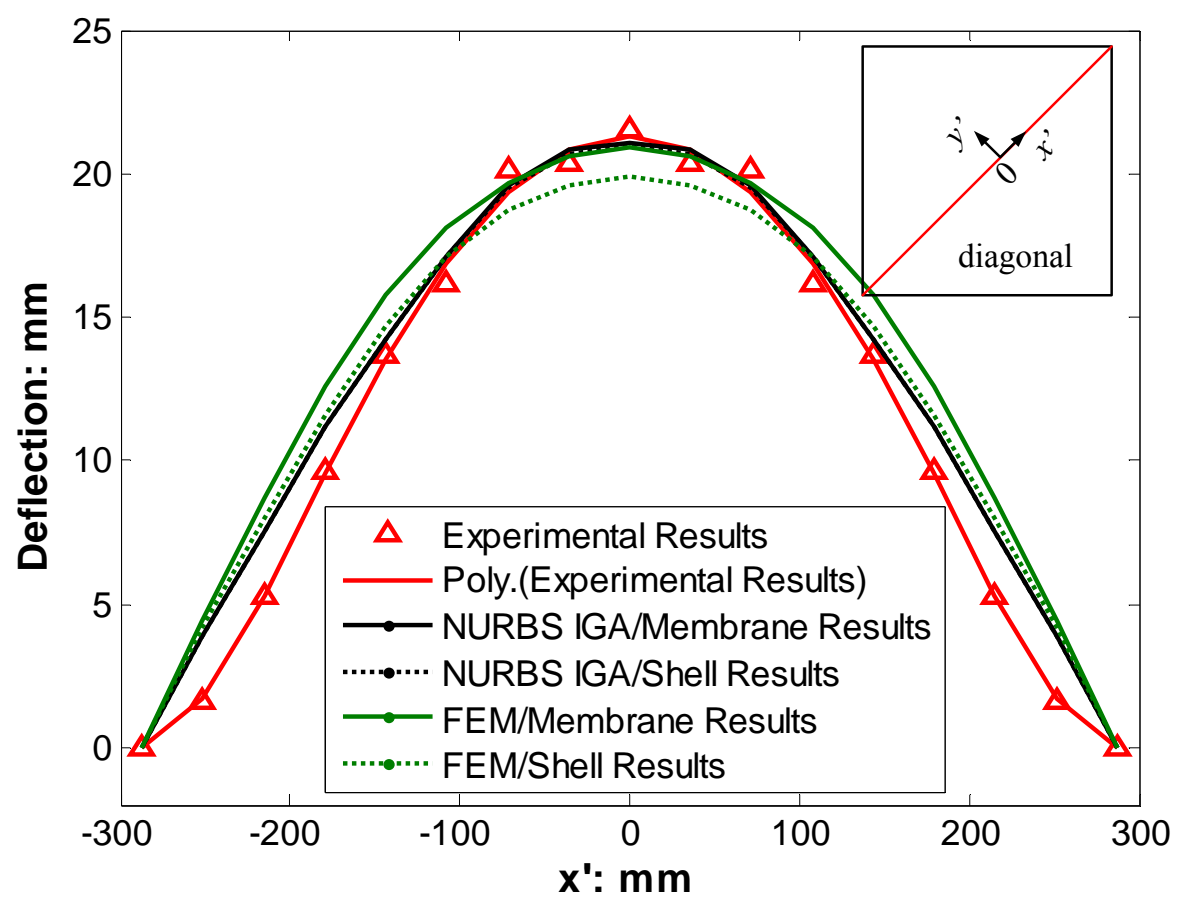

(a)

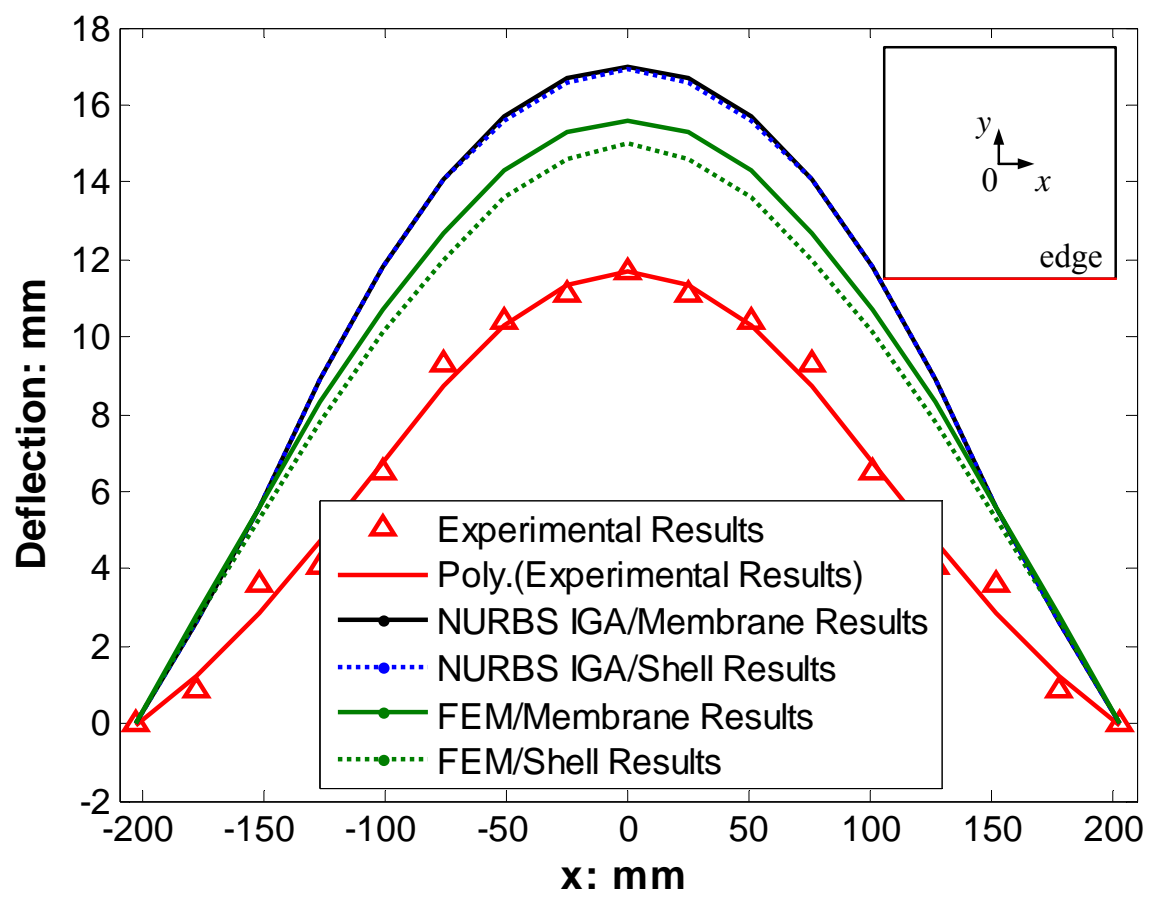

(b)

Figure 8. Square membrane under static pressure load: (a) comparison of the deflections along the diagonal; (b) comparison of the deflections along the edge. 


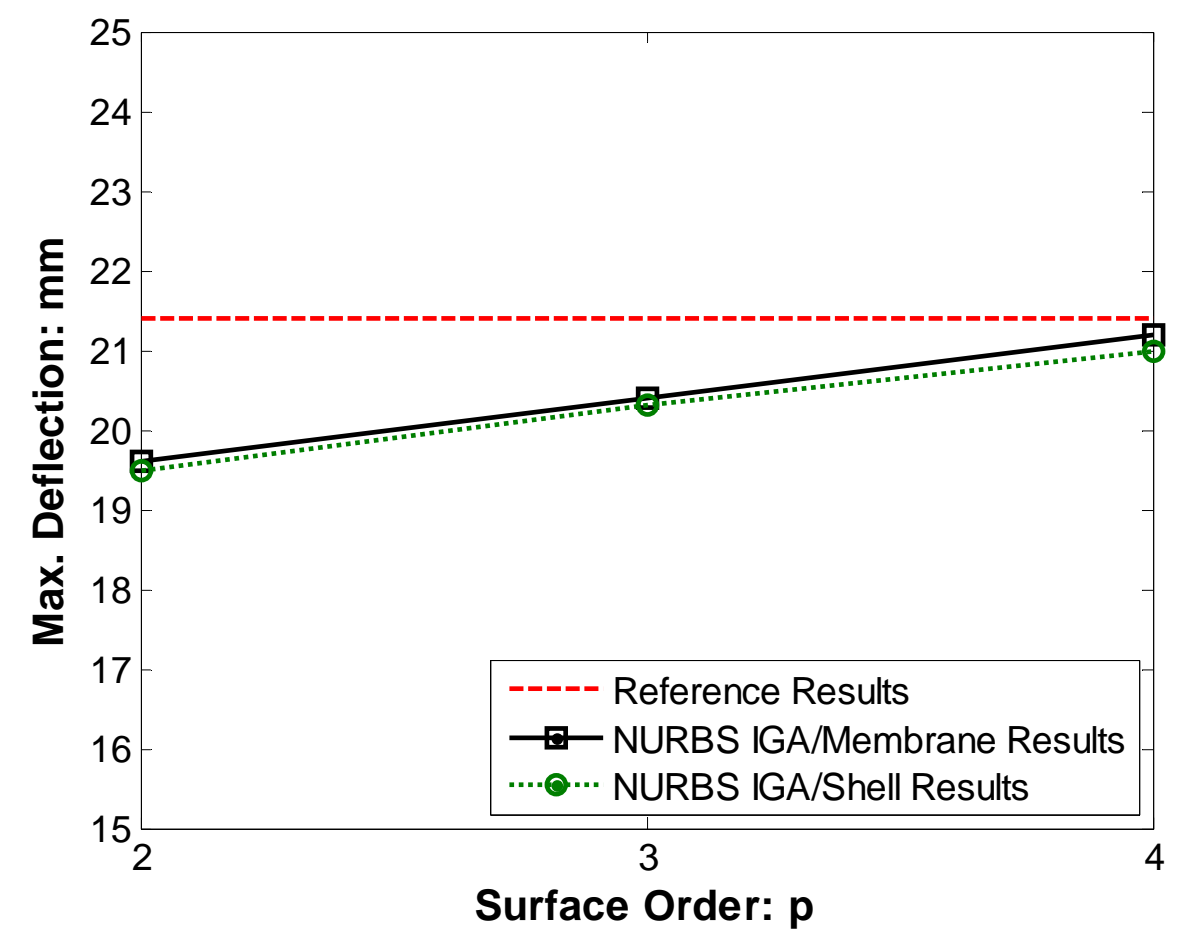

Figure 9. Square membrane under static pressure load: effect of surface order.

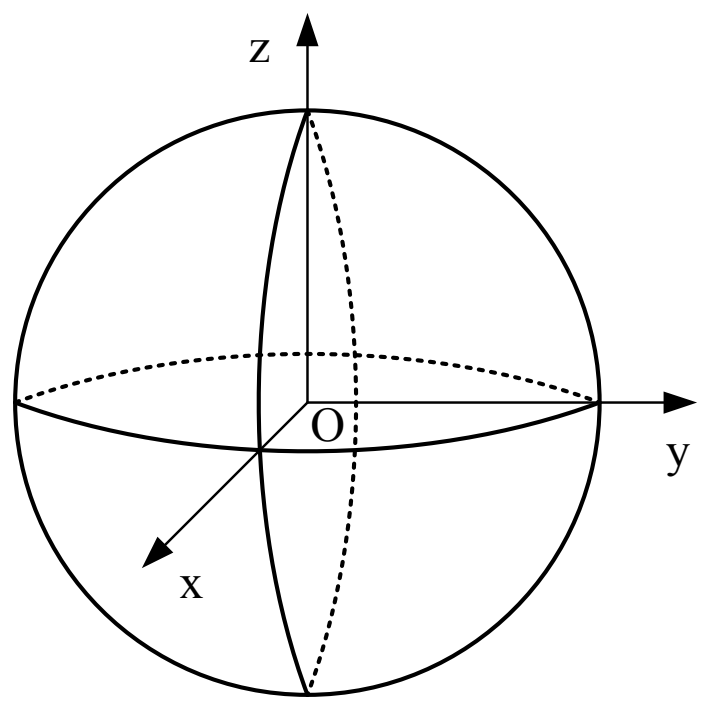

(a)

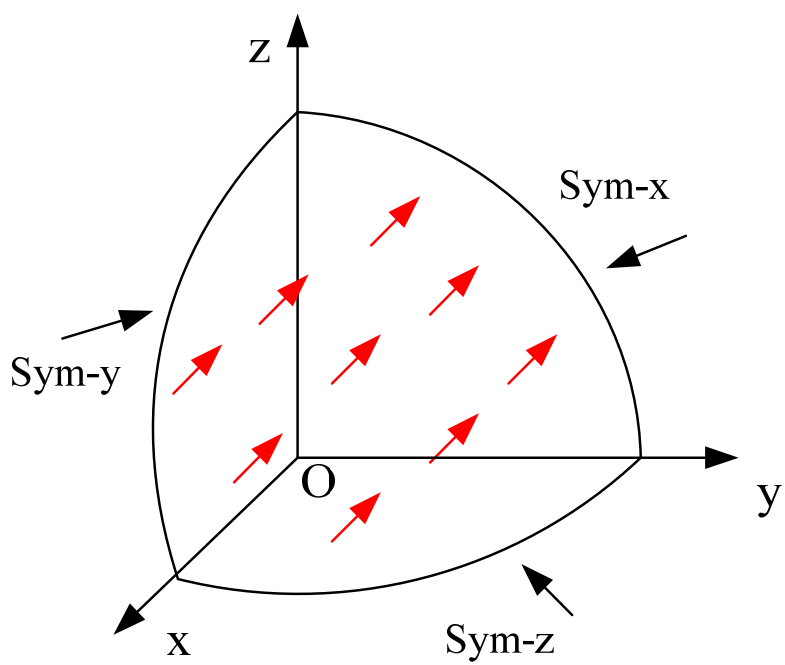

(b)

Figure 10. Problem setting of a spherical membrane structure subjected to uniform internal pressure. Due to symmetry, only an eighth of the sphere is used in the isogeometric analyses. 

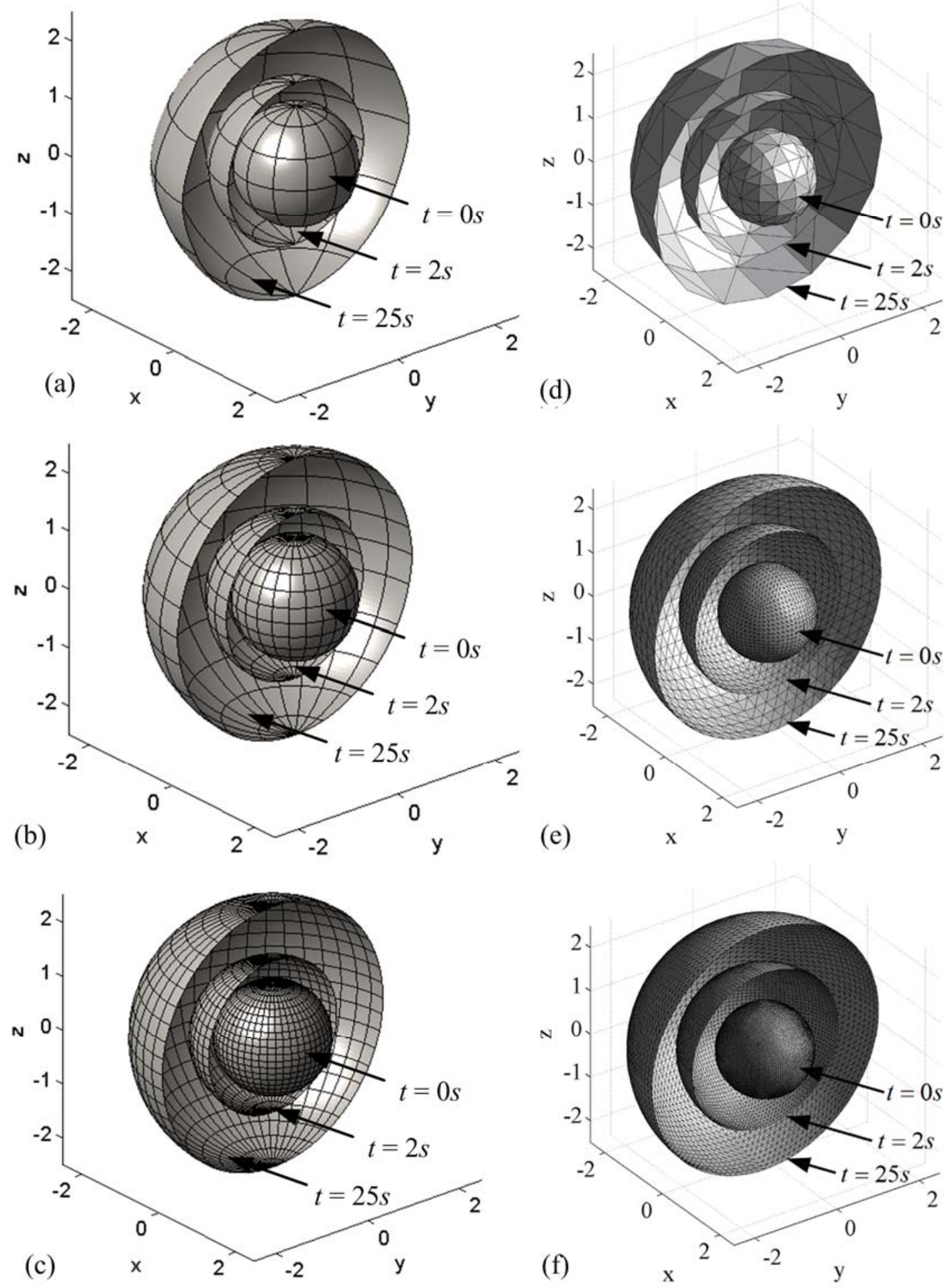

Figure 11. Initial and deformed configurations of the spherical membrane structure under uniform internal pressure solved by the NURBS IGA with (a) $3 \times 3$, (b) $6 \times 6$ and (c) $12 \times 12$ surface elements, and the FEM with (d) 192, (e) 3072 and (f) 49125 traditional Lagrangian 3-noded triangular elements. 


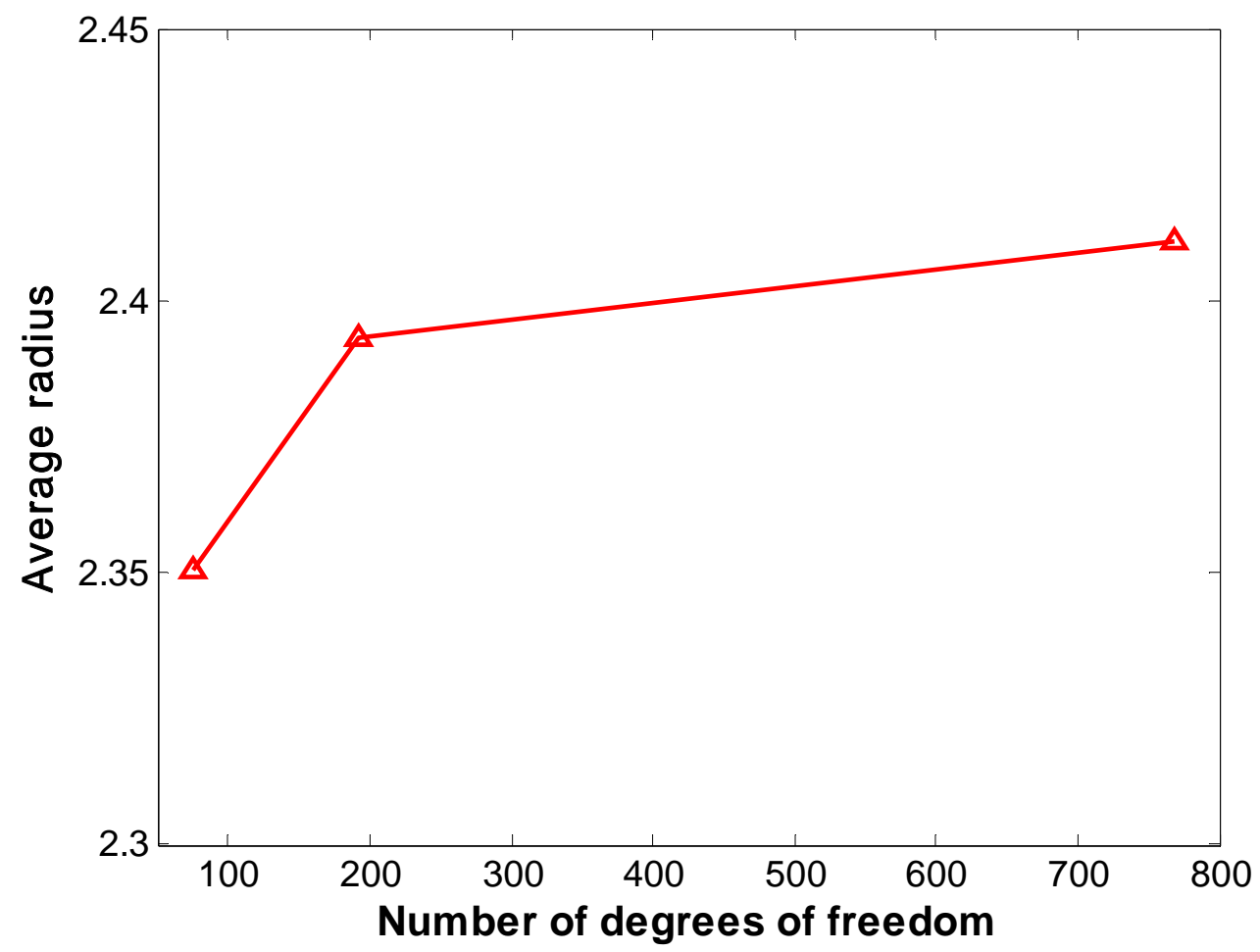

Figure 12. Spherical membrane under uniform internal pressure: convergence of average radius under as a function of the number of degrees of freedom ( $k$-refinement strategy).

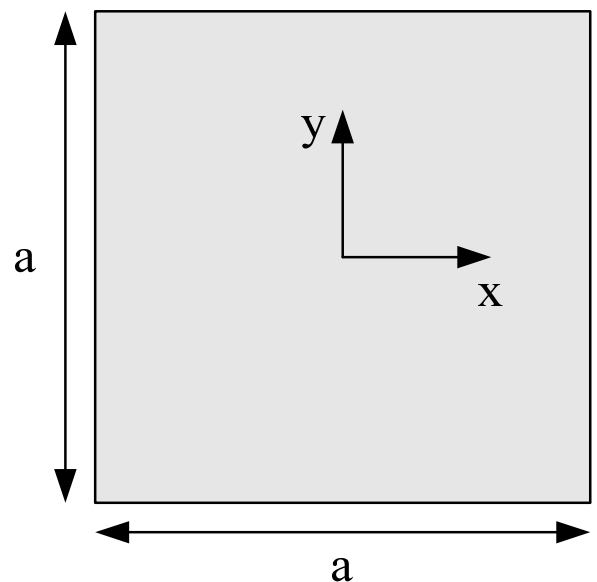

Figure 13. Problem setting of the inflation of a square airbag. 

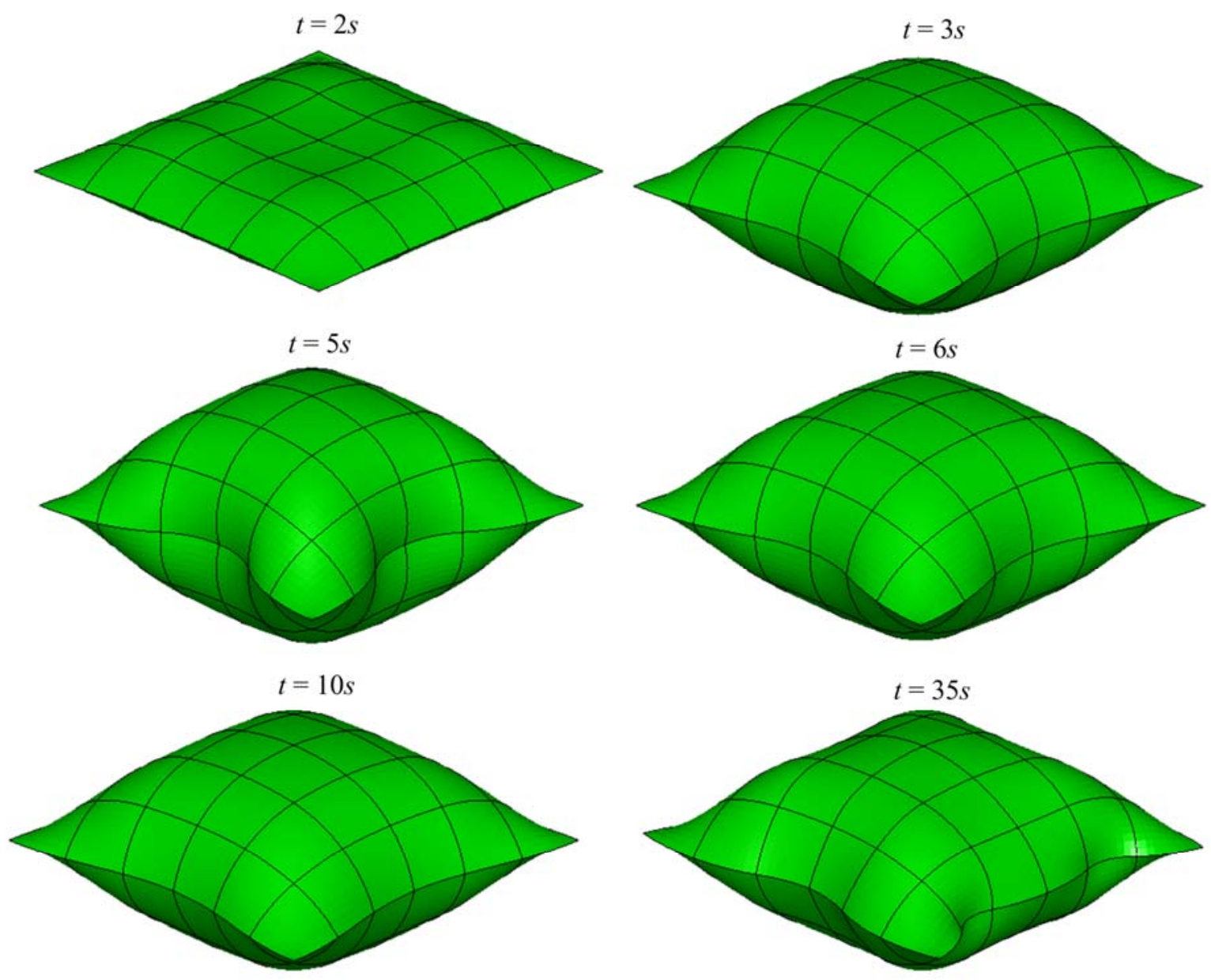

Figure 14. Square airbag: deformed shape histories solved by the IGA using $6 \times 6$ NURBS surface elements at time $2 \mathrm{~s}, 3 \mathrm{~s}, 5 \mathrm{~s}, 6 \mathrm{~s} 10 \mathrm{~s}$ and $35 \mathrm{~s}$. 

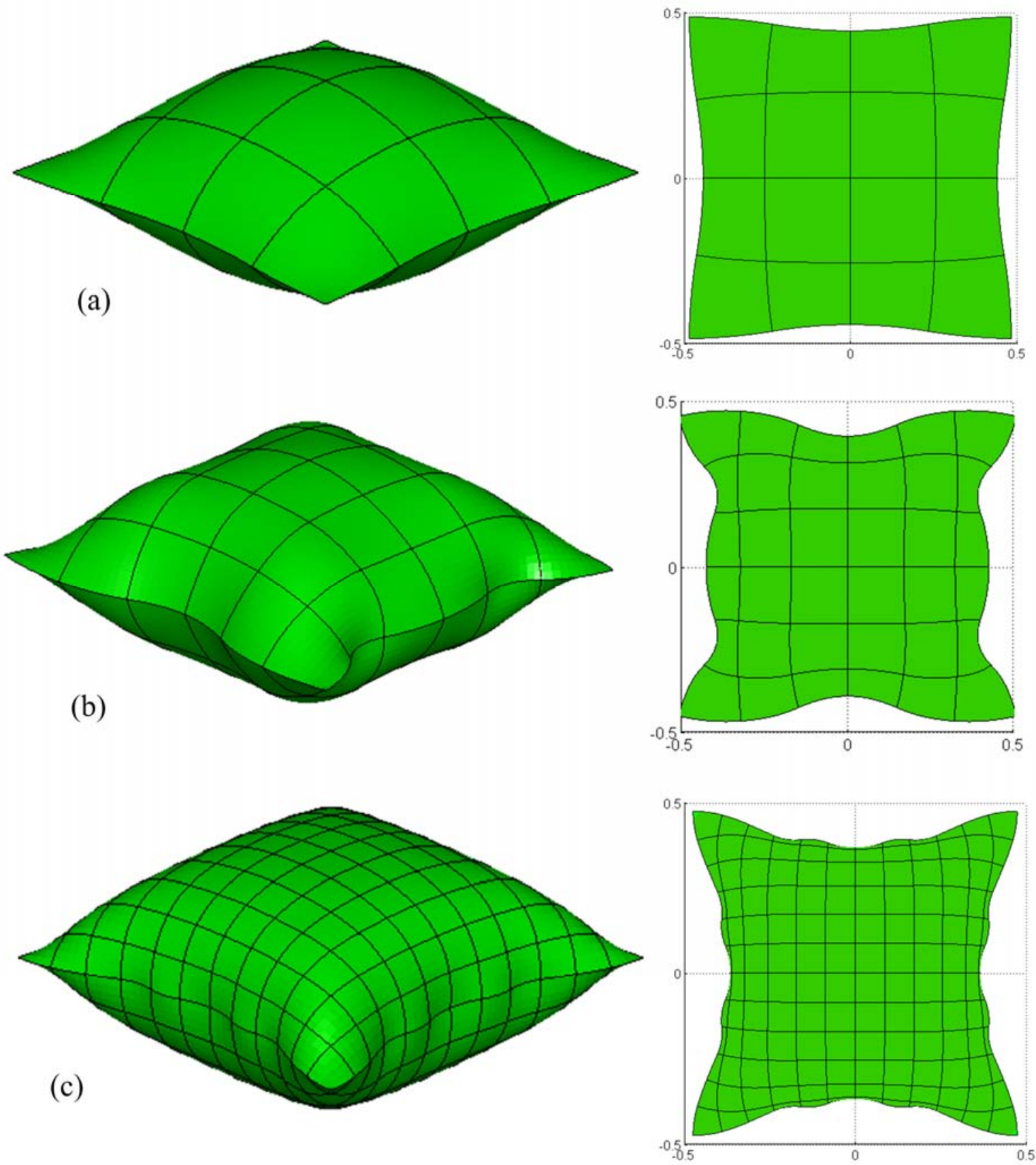

Figure 15. Square airbag: deformed quasi-static shapes solved by the IGA using (a) $4 \times 4$, (b) $6 \times 6$, (c) $12 \times 12$ NURBS surface elements. The left column shows a side view while the right column corresponds to the top view. 


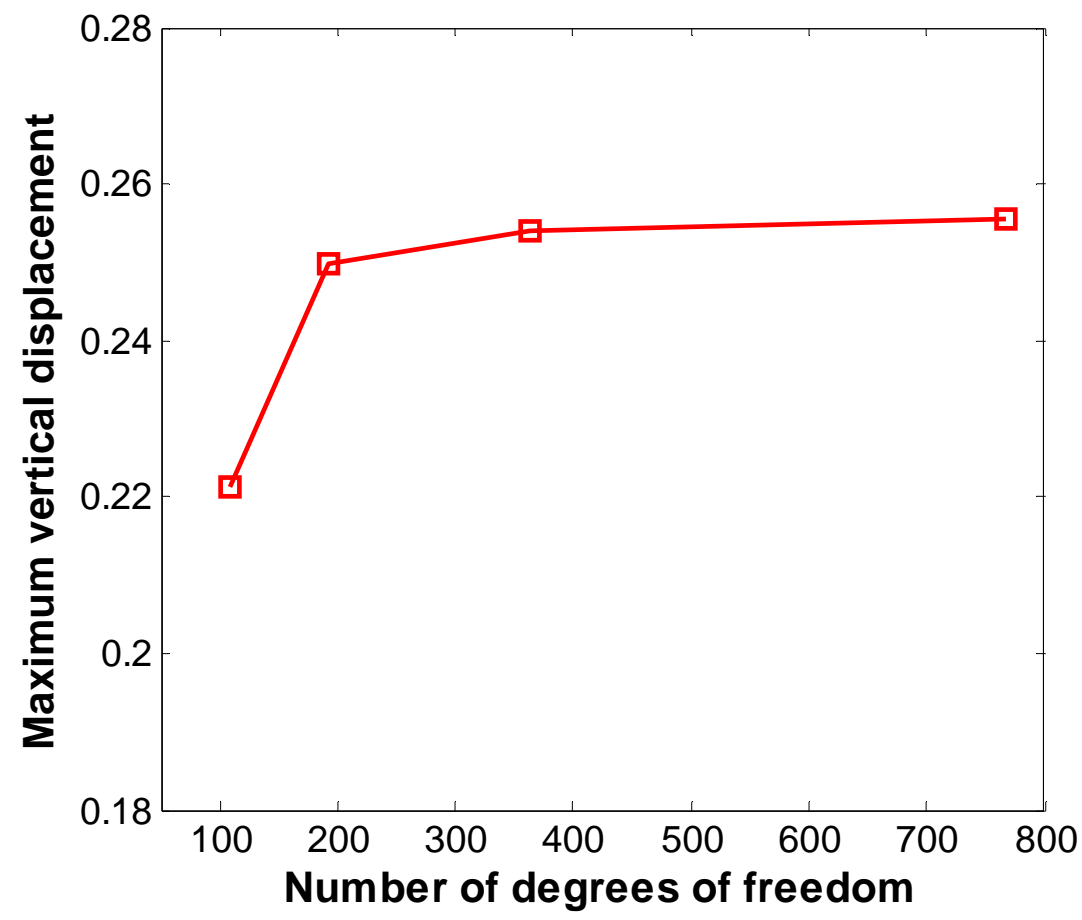

Figure 16. Square airbag: convergence of maximum vertical displacement as a function of the number of degrees of freedom ( $k$-refinement strategy).

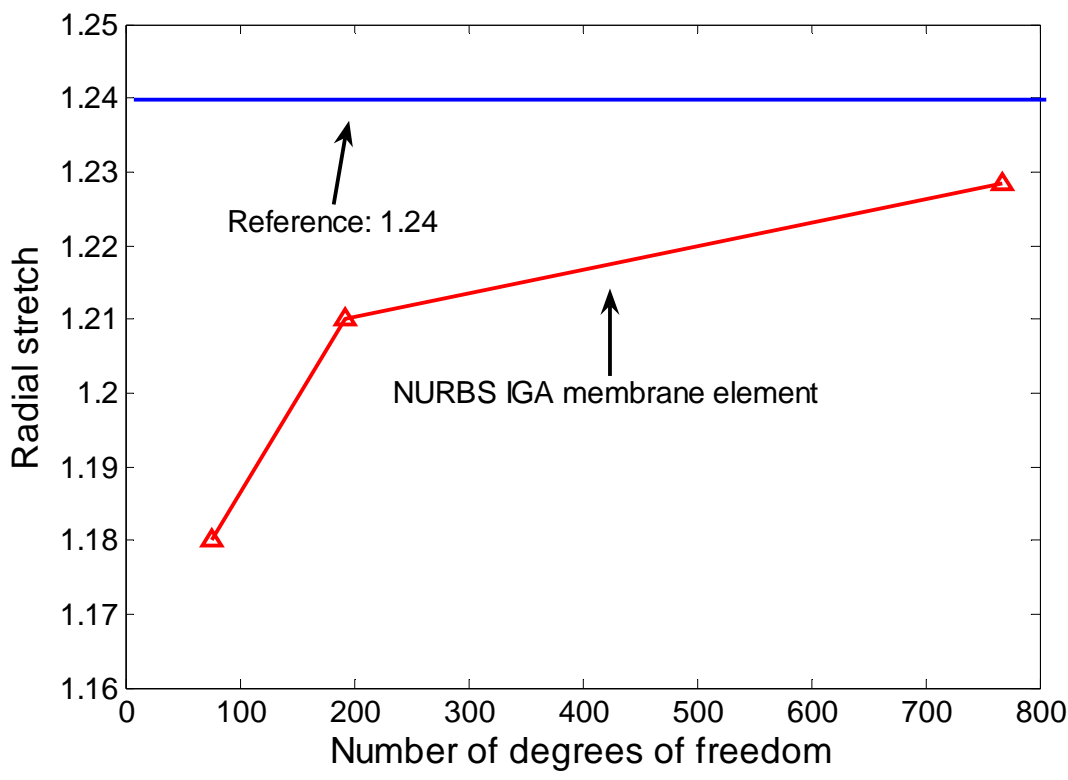

Figure 17. Hyperelastic balloon: convergence of radial stretch as a function of the number of degrees of freedom ( $k$-refinement strategy). 